\title{
Multiwavelength Study of High-Redshift Blazars
}

\author{
N. Sahakyan, ${ }^{1,2 \star}$ D. Israyelyan, ${ }^{1}$ G. Harutyunyan ${ }^{1}$ M. Khachatryan ${ }^{1}$ and S. Gasparyan ${ }^{1}$ \\ ${ }_{1}^{1}$ ICRANet Armenia, Marshall Baghramian Avenue 24a, Yerevan 0019, Republic of Armenia \\ ${ }^{2}$ ICRANet, Piazza della Repubblica 10, I-65122 Pescara, Italy
}

Accepted XXX. Received YYY; in original form ZZZ

\begin{abstract}
High-redshift blazars are among the most powerful objects in the Universe. The spectral and temporal properties of thirty-three distant blazars $(z>2.5)$ detected in the high energy $\gamma$-ray band are investigated by analyzing the Fermi-LAT and Swift UVOT/ XRT data. The considered sources have soft time averaged $\gamma$-ray spectra $\left(\Gamma_{\gamma} \geq 2.2\right)$ whereas those that have been observed in the X-ray band have hard X-ray spectra $\left(\Gamma_{\mathrm{X}}=1.01-1.86\right)$. The $\gamma$-ray flux of high-redshift blazars ranges from $4.84 \times 10^{-10}$ to $1.50 \times 10^{-7}$ photon $\mathrm{cm}^{-2} \mathrm{~s}^{-1}$ and the luminosity is within $(0.10-5.54) \times 10^{48} \mathrm{erg} \mathrm{s}^{-1}$ which during the $\gamma$-ray flares increases up to $(0.1-1) \times 10^{50} \mathrm{erg} \mathrm{s}^{-1}$. In the X-ray band, only the emission of PKS 0438-43, B2 $0743+25$ and TXS $0222+185$ is found to vary in different Swift XRT observations whereas in the $\gamma$-ray band, the emission is variable for fourteen sources: the flux of B3 $1343+451$ and PKS 0537-286 changes in sub-day scales, that of PKS 0347-211 and PKS 0451-28 in day scales, while the $\gamma$-ray variability of the others is in week or month scales. The properties of distant blazar jets are derived by modeling the multiwavelength spectral energy distributions within a one-zone leptonic scenario assuming that the X-ray and $\gamma$-ray emissions are produced from inverse Compton scattering of synchrotron and dusty torus photons. From the fitting, the emission region size is found to be $\leq 0.05 \mathrm{pc}$ and the magnetic field and the Doppler factor are correspondingly within $0.10-1.74 \mathrm{G}$ and $10.0-27.4$. By modeling the optical-UV excess, we found that the central black hole masses and accretion disk luminosities are within $L_{\mathrm{d}} \simeq(1.09-10.94) \times 10^{46} \mathrm{erg} \mathrm{s}^{-1}$ and $(1.69-5.35) \times 10^{9} M_{\odot}$, respectively.
\end{abstract}

Key words: Galaxies: active-Gamma rays: galaxies-radiation mechanisms: non-thermal

\section{INTRODUCTION}

Blazars are one of the most luminous objects in the Universe. In the unification scheme of radio-loud active galactic nuclei (AGNs) blazars are a subclass with a relativistic jet making a small angle (a few degrees) with the observer's line of sight (Urry \& Padovani 1995). The jets are sources of non-thermal emission which cover the entire electromagnetic spectrum, from radio to high energy (HE; $>100 \mathrm{MeV}$ ) or very high energy (VHE; > $100 \mathrm{GeV}$ ) $\gamma$-ray bands (Padovani et al. 2017). This nonthermal emission is varying in short time scales (e.g., in minute scales in the $\gamma$-ray band Ackermann et al. 2016; Foschini et al. 2011, 2013; Nalewajko 2013; Brown 2013; Rani et al. 2013a; Saito et al. 2013; Hayashida et al. 2015a) with a substantial increase in luminosity. The flux variation as well as the observed superluminal motion, the high degree of polarization and other observed features can be explained by the relativistic beaming effects.

Blazars are usually grouped into two large classes based on the absence or presence of emission lines in their optical spectra, i.e., BL Lacertae objects (BL Lacs) have weak or no emission lines;

^ E-mail: narek@icra.it the equivalent width (EW) of the emission line $<5 \AA$ in the rest frame, while the flat-spectrum radio quasars (FSRQs) have stronger emission lines (EW > $5 \AA$ ) (Urry \& Padovani 1995). Based on the position of the synchrotron peak in the rest frame $\left(\nu_{\mathrm{p}}^{\text {syn }}\right)$, blazars are further classified as low-synchrotron-peaked (LSP for $v_{\mathrm{p}}^{\text {syn }}<10^{14}$ $\mathrm{Hz}$ ), intermediate-synchrotron-peaked (ISP for $10^{14}<v_{\mathrm{p}}^{\text {syn }}<10^{15}$ $\mathrm{Hz}$ ), and high-synchrotron-peaked (HSP for $v_{\mathrm{p}}^{\mathrm{syn}}>10^{15} \mathrm{~Hz}$ ) objects (Padovani \& Giommi 1995; Abdo et al. 2010a).

The broadband spectral energy distribution (SED) of blazars shows a typical double-peaked structure. The low-energy peak (extending from radio to UV/soft X-rays) is produced by synchrotron emission from the relativistic electrons in the jet. The HE component (above X-ray band) is often attributed to Inverse Compton (IC) scattering of photons produced either inside (synchrotronself Compton, (SSC); Ghisellini et al. 1985; Bloom \& Marscher 1996; Maraschi et al. 1992) or outside of the jet (external inverse Compton, (EIC); Błażejowski et al. 2000; Sikora et al. 1994; Ghisellini \& Tavecchio 2009). The external photons can be from the accretion disk, broad-line region and dusty torus surrounding the disc. In alternative models, the HE component is due to the interaction of protons accelerated along with electrons in the jet (e.g., 
Table 1. List of $\gamma$-ray emitting blazars at $z>2.5$. The results of observation of distant blazars from August 4, 2008, to August 4, 2018 by Fermi-LAT are presented in the right part of the table.

\begin{tabular}{|c|c|c|c|c|c|c|c|c|c|c|}
\hline Object & 4FGL name & Class & $\overline{\mathrm{RA}}$ & $\overline{\text { Dec }}$ & $\overline{F_{\gamma}{ }^{\mathrm{T}}}$ & Photon index & $\overline{T S}$ & $E_{\max }^{2}$ & Probability & $\bar{z}$ \\
\hline GB $1508+5714$ & $\mathrm{~J} 1510.1+5702$ & FSRQ & 227.54 & 57.04 & $0.76 \pm 0.15$ & $2.95 \pm 0.15$ & 63.50 & 8.76 & 0.8268 & 4.31 \\
\hline PKS 1351-018 & J1354.3-0206 & FSRQ & 208.50 & -2.11 & $1.11 \pm 0.15$ & $2.69 \pm 0.09$ & 98.49 & 1.30 & 0.7010 & 3.72 \\
\hline MG3 J163554+3629 & $\mathrm{J} 1635.6+3628$ & $\mathrm{BCU}$ & 248.92 & 36.48 & $1.46 \pm 0.27$ & $2.84 \pm 0.12$ & 123.95 & 3.74 & 0.8413 & 3.65 \\
\hline NVSS J121915 +365718 & $\mathrm{J} 1219.0+3653$ & $\mathrm{BCU}$ & 184.77 & 36.89 & $0.22 \pm 0.08$ & $2.20 \pm 0.14$ & 40.56 & 2.67 & 0.8273 & 3.53 \\
\hline PKS 0335-122 & J0337.8-1157 & FSRQ & 54.47 & -11.96 & $0.72 \pm 0.16$ & $2.69 \pm 0.13$ & 49.47 & 10.19 & 0.9245 & 3.44 \\
\hline PKS 0537-286 & J0539.9-2839 & FSRQ & 84.99 & -28.66 & $4.38 \pm 0.18$ & $2.72 \pm 0.03$ & 1694.80 & 7.60 & 0.9619 & 3.10 \\
\hline TXS 0800+618 & J0805.4+6147 & FSRQ & 121.36 & 61.80 & $2.23 \pm 0.14$ & $2.82 \pm 0.05$ & 475.17 & 4.83 & 0.8441 & 3.03 \\
\hline S4 $1427+543$ & $\mathrm{~J} 1428.9+5406$ & FSRQ & 217.23 & 54.11 & $0.59 \pm 0.15$ & $2.62 \pm 0.14$ & 67.10 & 10.26 & 0.7831 & 3.01 \\
\hline GB6 J0733+0456 & J0733.8+0455 & FSRQ & 113.47 & 4.93 & $1.14 \pm 0.15$ & $2.39 \pm 0.07$ & 197.26 & 17.65 & 0.9854 & 3.01 \\
\hline B2 $0743+25$ & $\mathrm{~J} 0746.4+2546$ & FSRQ & 116.60 & 25.77 & $2.06 \pm 0.19$ & $2.87 \pm 0.07$ & 280.99 & 1.52 & 0.7449 & 2.99 \\
\hline PKS 0347-211 & J0349.8-2103 & FSRQ & 57.47 & -21.06 & $3.25 \pm 0.15$ & $2.47 \pm 0.03$ & 1372.81 & 5.83 & 0.9690 & 2.94 \\
\hline S4 1124+57 & $\mathrm{J} 1127.4+5648$ & FSRQ & 171.87 & 56.80 & $0.96 \pm 0.13$ & $2.75 \pm 0.09$ & 147.30 & 7.09 & 0.8495 & 2.89 \\
\hline MRSS 291-081526 & J2313.9-4501 & $\mathrm{BCU}$ & 348.49 & -45.02 & $0.89 \pm 0.20$ & $2.80 \pm 0.13$ & 81.12 & 2.25 & 0.7550 & 2.88 \\
\hline PKS 0438-43 & J0440.3-4333 & FSRQ & 70.09 & -43.55 & $2.24 \pm 0.20$ & $2.60 \pm 0.05$ & 574.84 & 2.00 & 0.9225 & 2.85 \\
\hline S4 2015+65 & $\mathrm{J} 2015.4+6556$ & FSRQ & 303.86 & 65.95 & $0.39 \pm 0.15$ & $2.37 \pm 0.15$ & 23.77 & 4.34 & 0.7257 & 2.84 \\
\hline $87 G B 214302.1+095227$ & $\mathrm{~J} 2145.5+1006$ & BL Lac & 326.38 & 10.12 & $0.048 \pm 0.014$ & $1.71 \pm 0.19$ & 40.46 & 67.15 & 0.9874 & 2.83 \\
\hline MG2 J174803+3403 & $\mathrm{J} 1748.0+3403$ & FSRQ & 267.01 & 34.06 & $0.97 \pm 0.13$ & $2.31 \pm 0.07$ & 284.21 & 31.63 & 0.9934 & 2.76 \\
\hline PKS 0834-20 & J0836.5-2026 & FSRQ & 129.13 & -20.45 & $1.93 \pm 0.19$ & $2.94 \pm 0.08$ & 171.15 & 1.15 & 0.5796 & 2.75 \\
\hline TXS $0222+185$ & J0224.9+1843 & FSRQ & 36.24 & 18.72 & $1.72 \pm 0.22$ & $3.05 \pm 0.12$ & 101.08 & 2.59 & 0.5327 & 2.69 \\
\hline OD 166 & J0242.3+1102 & FSRQ & 40.59 & 11.05 & $1.94 \pm 0.20$ & $2.59 \pm 0.06$ & 252.83 & 6.91 & 0.8651 & 2.68 \\
\hline $\begin{array}{l}\text { CRATES J233930 } \\
+024420\end{array}$ & $\mathrm{~J} 2339.6+0242$ & $\mathrm{BCU}$ & 354.90 & 2.71 & $1.00 \pm 0.21$ & $2.58 \pm 0.11$ & 94.50 & 6.17 & 0.8537 & 2.66 \\
\hline TXS 0907+230 & J0910.6+2247 & FSRQ & 137.67 & 22.80 & $1.17 \pm 0.14$ & $2.37 \pm 0.06$ & 262.58 & 5.38 & 0.9578 & 2.66 \\
\hline PMN J1441-1523 & J1441.6-1522 & FSRQ & 220.41 & -15.38 & $0.65 \pm 0.23$ & $2.32 \pm 0.13$ & 72.02 & 11.77 & 0.9535 & 2.64 \\
\hline $\begin{array}{l}\text { CRATES J105433 } \\
+392803\end{array}$ & $\mathrm{~J} 1054.2+3926$ & $\mathrm{BCU}$ & 163.56 & 39.44 & $0.27 \pm 0.09$ & $2.30 \pm 0.16$ & 34.05 & 4.79 & 0.9000 & 2.63 \\
\hline MG1 J154930+1708 & $\mathrm{J} 1549.6+1710$ & BL Lac & 237.41 & 17.18 & $0.17 \pm 0.08$ & $2.01 \pm 0.16$ & 44.75 & 17.03 & 0.9759 & 2.62 \\
\hline TXS 1448+093 & $\mathrm{J} 1450.4+0910$ & FSRQ & 222.62 & 9.18 & $0.76 \pm 0.13$ & $2.35 \pm 0.08$ & 130.24 & 10.58 & 0.9599 & 2.61 \\
\hline PMN J0226+0937 & $\mathrm{J} 0226.5+0938$ & FSRQ & 36.63 & 9.63 & $0.48 \pm 0.12$ & $2.18 \pm 0.10$ & 96.49 & 56.42 & 0.9523 & 2.61 \\
\hline PKS 0451-28 & J0453.1-2806 & FSRQ & 73.29 & -28.11 & $5.83 \pm 0.17$ & $2.66 \pm 0.02$ & 3118.20 & 10.77 & 0.9815 & 2.56 \\
\hline B3 0908+416B & J0912.2+4127 & FSRQ & 138.06 & 41.46 & $1.51 \pm 0.14$ & $2.42 \pm 0.05$ & 539.57 & 12.10 & 0.9903 & 2.56 \\
\hline TXS 1616+517 & $\mathrm{J} 1618.0+5139$ & FSRQ & 244.52 & 51.67 & $0.69 \pm 0.13$ & $2.68 \pm 0.12$ & 72.65 & 12.38 & 0.8408 & 2.56 \\
\hline B3 $1624+414$ & $\mathrm{~J} 1625.7+4134$ & FSRQ & 246.45 & 41.57 & $1.38 \pm 0.14$ & $2.49 \pm 0.06$ & 395.12 & 9.69 & 0.9787 & 2.55 \\
\hline B3 $1343+451$ & $\mathrm{~J} 1345.5+4453 \mathrm{c}$ & FSRQ & 206.39 & 44.88 & $15.01 \pm 0.16$ & $2.25 \pm 0.008$ & 34652.79 & 24.25 & 0.9994 & 2.53 \\
\hline PKS 2107-105 & $\mathrm{J} 2110.2-1021 \mathrm{c}$ & FSRQ & 317.56 & -10.36 & $0.88 \pm 0.18$ & $2.66 \pm 0.13$ & 53.29 & 5.46 & 0.6500 & 2.50 \\
\hline
\end{tabular}

${ }^{1}$ Integrated $\gamma$-ray flux in units of $\times 10^{-8}$ photon $\mathrm{cm}^{-2} \mathrm{~s}^{-1}$

2 Photon energy in GeV.

Table 2. Spectral parameters of the sources modeled with logparabola.

\begin{tabular}{lccc}
\hline \hline Object & $F_{\gamma}{ }^{1}$ & $\alpha$ & $\beta$ \\
\hline PKS 1351-018 & $(6.86 \pm 1.66) \times 10^{-9}$ & $2.20 \pm 0.23$ & $0.63 \pm 0.23$ \\
PKS 0537-286 & $(4.15 \pm 0.20) \times 10^{-8}$ & $2.66 \pm 0.04$ & $0.10 \pm 0.03$ \\
TXS 0800+618 & $(2.04 \pm 0.16) \times 10^{-8}$ & $2.67 \pm 0.08$ & $0.17 \pm 0.06$ \\
B2 0743+25 & $(1.72 \pm 0.23) \times 10^{-8}$ & $2.53 \pm 0.14$ & $0.40 \pm 0.13$ \\
PKS 0347-211 & $(2.69 \pm 0.17) \times 10^{-8}$ & $2.32 \pm 0.05$ & $0.19 \pm 0.03$ \\
PKS 0438-43 & $(1.15 \pm 0.23) \times 10^{-8}$ & $2.35 \pm 0.12$ & $0.48 \pm 0.11$ \\
S4 2015+65 & $(1.66 \pm 1.00) \times 10^{-9}$ & $2.42 \pm 0.35$ & $0.42 \pm 0.31$ \\
PKS 0834-20 & $(1.76 \pm 0.20) \times 10^{-8}$ & $2.62 \pm 0.16$ & $0.29 \pm 0.13$ \\
OD 166 & $(1.45 \pm 0.24) \times 10^{-8}$ & $2.43 \pm 0.11$ & $0.24 \pm 0.08$ \\
TXS 0907+230 & $(8.68 \pm 1.77) \times 10^{-8}$ & $2.28 \pm 0.09$ & $0.12 \pm 0.06$ \\
PKS 0451-28 & $(5.56 \pm 0.18) \times 10^{-8}$ & $2.56 \pm 0.04$ & $0.09 \pm 0.02$ \\
B3 1343+451 & $(1.41 \pm 0.02) \times 10^{-7}$ & $2.18 \pm 0.01$ & $0.07 \pm 0.006$ \\
\hline
\end{tabular}

Böttcher et al. 2013). In this case, the blazars also can emit VHE neutrinos (e.g., Ansoldi et al. 2018; Gao et al. 2019; Cerruti et al. 2019; Keivani et al. 2018; Liao et al. 2018; Sahakyan 2018, 2019; Wang et al. 2018; Righi et al. 2019).

Blazars are the dominant sources in the extragalactic $\gamma$-ray sky. Among the total 5000 sources in the Fermi Large Area Telescope (Fermi-LAT) fourth source catalog of $\gamma$-ray sources (4FGL;
Abdollahi et al. 2020), 2800 are blazars, 45 are radio galaxies, and 19 are other AGNs. Low to high redshift blazars are observed showing different redshift distributions for FSRQs and BL Lacs; the distribution of FSRQs has a peak around $z=1$ with a median of $1.14 \pm 0.62$ and there is a high number of FSRQs at the redshift of $\simeq 0.5-2.0$, while the peak of BL Lacs is at $z=0.3$ with a mean of $0.34 \pm 0.42$. There are only 105 sources detected with $z>2(3.75$ $\%$ of total sources) and only 33 with $z>2.5(1.18 \%)$.

Blazars harboring supermassive black holes are valuable sources for studying the relativistic outflows and formation and propagation of relativistic jets. In this context, the high redshift blazars $(z>2.5)$ are of particular interest; they are the most powerful nonexplosive astrophysical sources having ever been detected in the $\gamma$-ray band. Their study can shed light on the further understanding of the cosmological evolution of blazars and supermassive black holes and also on the evolution of relativistic jets across different cosmic epochs (Volonteri 2010). Moreover, the $\gamma$-ray observations of distant blazars are important since a limit on the density of the Extragalactic Background Light (EBL) can be derived. The $\gamma$-rays , as they propagate from their sources to the Earth, are subject to absorption through two-photon pair production when interacting with EBL photons (e.g., Stecker et al. 2006; Domínguez et al. 2011). This absorption feature is visible in the spectrum of the 
nearby sources only at VHEs while for distant sources $(z>2.0)$, it can be significant already at tens of GeV. Thus, the EBL density in a redshift-dependence way can be constrained or measured by analyzing the $\gamma$-ray data (e.g., Abdo et al. 2010c; Ackermann et al. 2012; Fermi-LAT Collaboration et al. 2018; Desai et al. 2019). However, if the $\gamma$-ray spectrum does not extend above $10 \mathrm{GeV}$ to constrain the EBL density, by the theoretical interpretation of the data, the intrinsic source processes can be investigated and separated from the propagation effects which can be a help in the observations of distant blazars by future telescopes (e.g., CTA).

The vast majority of high redshift blazars are LSPs so the HE peak in their SEDs is at MeV below the Fermi-LAT band. They are sometimes also called 'MeV' blazars (Bloemen et al. 1995), being bright and strong X-ray emitters. In the X-ray band, these sources usually have a hard spectrum which corresponds to the rising part of the HE component, so these data are crucial for investigation of the origin of nonthermal emission (Ghisellini et al. 2010b). Yet, due to the shift of SED peaks, for some blazars the direct thermal emission from the accretion disc is visible in the optical-UV band (the big blue bump) which can be modeled to derive the accretion disc luminosity (Shakura \& Sunyaev 1973) and the black hole mass and so to understand the properties of the central source. So, the Neil Gehrels Swift Observatory (Gehrels et al. (2004), hereafter Swift), taking the data in Optical/UV and X-ray bands, is an ideal instrument (within its sensitivity limit) for observing distant blazars.

Even though in the $\gamma$-ray band the distant blazars are relatively faint as compared with the X-ray band, Fermi-LAT observations are still crucial. The $\gamma$-ray band corresponds to the falling part of the HE component which combined with the X-ray data will fully constrain the second peak in the SED. This is fundamental allowing to derive the physical parameters of the jets. The multiwavelength observations and theoretical interpretations are a regular approach and a unique way of probing the physical condition of the plasma in the jet. From the theoretical modeling point of view, distant blazars are excellent sources for studying accretion disc-jet connection in the early epoch of quasar formation as well as for probing the environments around supermassive black holes.

Identification of distant blazars and their investigation has always been one of the actively discussed topics in the blazar research (e.g., Paliya et al. 2016; Paliya 2015; Ghisellini et al. 2011; Li et al. 2018; Ajello et al. 2016; Kaur et al. 2017; Ackermann et al. 2017; Kaur et al. 2018; Marcotulli et al. 2017; Paliya et al. 2019). In contrast to nearby blazars, good quality multiwavelength data are missing for the high redshift blazars, which significantly complicates their detailed study. However, due to improved sensitivity of the instruments (e.g., X-ray and $\gamma$-ray observatories) and wide-field surveys in the low energy bands (e.g., Sloan Digital Sky Survey (York et al. 2000) or WISE Wright et al. (2010)), the number of high redshift blazars with sufficient multiwavelength data has been significantly increased. Along with continuous $\gamma$-ray observations of some distant blazars since 2008 this opens new perspectives for exploring the physics of distant blazars.

Motivated by the large number of detected high redshift $\gamma$-ray emitting blazars, with the aim to characterize their multiwavelength emission properties, we perform an intense broadband study of all the thirty-three known $\gamma$-ray blazars beyond redshift 2.5. Using the improved Pass 8 dataset which is more suitable for studying weak sources, we perform a detailed spectral and temporal analysis of Fermi-LAT $\gamma$-ray data accumulated during 2008-2018. The $\gamma$-ray flux variation, not well explored for distant blazars, is investigated for the considered ten years, using an improved adaptive binning method. To characterize the physical properties of the considered sources in the X-ray and optical/UV bands, the data from their observation with both Swift X-ray Telescope (XRT) and Ultraviolet and Optical Telescope (UVOT) in the previous fifteen years are analyzed. This allows to collect unprecedented data in the optical/UV, $\mathrm{X}$-ray and $\gamma$-ray bands, which is used to constrain the multiwavelength SEDs. Then, through theoretical modeling of these SEDs, the physical parameters characterizing the sources (disc luminosity, black hole mass, etc.) and their jets (e.g., the distribution of underlying electrons, magnetic field, power, etc.) are derived, allowing a quantitative discussion and investigation of the state of plasma in these powerful jets. Taking into account the large number of the considered sources (thirty-three) and the amount of analyzed data, this is one of the most comprehensive studies of most distant and powerful blazars.

The paper is organized as follows. In Section 2, the list of high redshift blazars is presented. The Fermi-LAT and Swift data extraction and analyses are described in Sections 3 and 4, respectively. The data analysis results are presented in Section 5 and the origin of the multiwavelength emission is discussed in Section 6. The discussion and conclusion are given in Section 7 and a summary in Section 8.

\section{THE SAMPLE}

The fourth catalog of AGNs detected by Fermi-LAT contains more than 2863 AGNs detected above the $5 \sigma$ limit (Abdollahi et al. 2020). A small fraction of them (1.18\%) are distant blazars $z>2.5(\sim 20.7$ Gpc), including twenty-six FSRQs, two BL Lacs and five blazars of uncertain type (BCU). The coordinates, redshift and synchrotron peak classification of these sources are given in Table 1 . The most distant source at $z=4.31$ is the FSRQ GB $1508+5714$, whereas the BL Lacs 87 GB 214302.1+095227 and MG1 J154930+1708 are at $z=2.83$ and $z=2.62$, respectively, which is interesting, since due to low $\gamma$-ray luminosity BL Lacs are rarely observed at these distances. In this paper, the data collected by Fermi-LAT and Swift are analyzed to study the multiwavelength emission from high redshift blazars $(z>2.5)$ selected from the fourth catalog of AGNs detected by Fermi-LAT which are presented in Table 1. Throughout this paper, we assume the following standard cosmological parameters of $H_{0}=71 \mathrm{~km} \mathrm{~s}^{-1} \mathrm{Mpc}^{-1}$ and $\Omega_{\Lambda}=0.730$ (Freedman et al. 2001).

\section{FERMI-LAT OBSERVATIONS}

The Fermi-LAT data used in the current study had been accumulated during the first ten years of operation, from 4 August 2008 to 4 August 2018. The PASS 8 events from a circular region with a radius of $12^{\circ}$ around each source in the energy range from $100 \mathrm{MeV}$ to $500 \mathrm{GeV}$ were downloaded and analyzed with the Fermi Tools (1.2.1) using P8R3 instrument response functions. Good data and time intervals were selected using gtselect and gtmktime tools (with selection cuts "Event class $=128$ " and "evtype $=3$ ") using maximum zenith angle value of $90^{\circ}$ to avoid the $\gamma$-ray detection from the earth's limb. Using gtbin tool, the events are binned into $16.9^{\circ} \times 16.9^{\circ}$ a square region of interest (ROI) with pixels of $0.1^{\circ} \times 0.1^{\circ}$ and into 37 equal logarithmically spaced energy bins. The model file of each source (point-like sources included in the ROI and background) is generated using the 4FGL-DR2 version of the 4FGL which is based on 10 years of survey. The model file includes all 4FGL sources falling within the $\mathrm{ROI}+5^{\circ}$ region. The gll_iem_v07 and iso_P8R3_SOURCE_V2_v1 models are used to parameterize the 
Table 3. Swift XRT data analyses results. For the sources, when several observations were available, they were merged to estimate the averaged X-ray spectra. The sources for which the number of counts was enough to constrain the flux and the photon index in a single observation are marked with an asterisk (*).

\begin{tabular}{lcccc}
\hline \hline Object & $n_{H} \times 10^{20} \mathrm{~cm}^{-2}$ & $\Gamma_{\mathrm{X}}$ & $\log \left(\mathrm{F}_{(0.3-10) \mathrm{keV}) / \mathrm{erg} \mathrm{cm}^{-2} \mathrm{~s}^{-1}}\right.$ C-stat./dof \\
\hline GB 1508+5714 & 1.56 & $1.38 \pm 0.52$ & $-12.21 \pm 0.21$ & $0.58(20)$ \\
PKS 0537-286* & 2.20 & $1.17 \pm 0.04$ & $-11.42 \pm 0.02$ & $1.28(679)$ \\
TXS 0800+618* & 4.67 & $1.13 \pm 0.10$ & $-11.53 \pm 0.04$ & $1.08(355)$ \\
S4 1427+543 & 1.17 & $1.41 \pm 0.23$ & $-12.24 \pm 0.09$ & $1.1(102)$ \\
GB6 J0733+0456 & 7.72 & $1.65 \pm 0.65$ & $-12.80 \pm 0.23$ & $1.33(20)$ \\
B2 0743+25* & 4.50 & $1.15 \pm 0.03$ & $-11.30 \pm 0.01$ & $1.39(773)$ \\
PKS 0347-211 & 4.23 & $1.32 \pm 0.27$ & $-12.26 \pm 0.12$ & $1.24(75)$ \\
PKS 0438-43* & 1.41 & $1.25 \pm 0.09$ & $-11.52 \pm 0.04$ & $1.4(345)$ \\
S4 2015+65 & 10.6 & $1.79 \pm 0.69$ & $-12.28 \pm 0.23$ & $0.91(17)$ \\
MG2 J174803+3403 & 3.22 & $1.36 \pm 0.47$ & $-12.35 \pm 0.18$ & $0.61(33)$ \\
PKS 0834-20* & 6.07 & $1.07 \pm 0.09$ & $-11.67 \pm 0.04$ & $1.12(399)$ \\
TXS 0222+185* & 9.40 & $1.11 \pm 0.04$ & $-10.93 \pm 0.02$ & $1.89(684)$ \\
OD 166 & 8.97 & $1.86 \pm 0.40$ & $-12.37 \pm 0.15$ & $0.65(40)$ \\
TXS 0907+230 & 4.68 & $1.14 \pm 0.40$ & $-12.49 \pm 0.18$ & $1.26(40)$ \\
PMN J1441-1523 & 7.71 & $1.86 \pm 0.63$ & $-13.10 \pm 0.22$ & $0.51(25)$ \\
TXS 1448+093 & 2.11 & $2.33 \pm 0.62$ & $-13.30 \pm 0.16$ & $1.04(26)$ \\
PMN J0226+0937 & 6.57 & $1.49 \pm 0.37$ & $-12.83 \pm 0.15$ & $0.93(49)$ \\
PKS 0451-28* & 2.07 & $1.55 \pm 0.10$ & $-11.42 \pm 0.04$ & $0.99(297)$ \\
B3 0908+416B & 1.42 & $1.01 \pm 0.46$ & $-12.47 \pm 0.19$ & $1.11(30)$ \\
TXS 1616+517 & 1.98 & $1.25 \pm 0.33$ & $-12.77 \pm 0.14$ & $1.17(59)$ \\
B3 1343+451* & 1.78 & $1.21 \pm 0.17$ & $-12.22 \pm 0.07$ & $0.85(160)$ \\
PKS 2107-105 & 6.23 & $1.24 \pm 0.29$ & $-12.49 \pm 0.12$ & $1.67(72)$ \\
\hline
\end{tabular}

Galactic and extragalactic diffuse emission components. The spectral parameters set for the sources located within the ROI are allowed to be free in the analysis. The normalization parameters for the two diffuse components were also kept free.

The spectral analysis was performed using the binned maximumlikelihood method implemented in the gtlike tool. The source detection significance is estimated using the test statistic (TS) (Mattox et al. 1996) defined as $T S=2 \times\left(\log L_{1}-\log L_{0}\right)$ where $L_{1}$ is the likelihood of the data with a point source at the given position and $L_{0}$ without the source. The $\gamma$-ray spectral models of each considered source were assumed to be the same as in the 4FGL, and for those with a log-parabola an additional fit with the power-law model was performed. The spectra were calculated by separately running gtlike for smaller energy intervals equal in logarithmic space. Then, using the gtsrcprob tool and the model file obtained from the likelihood fitting, the energy of the highest-energy photon detected from the direction of each source is computed.

\section{$3.1 \gamma$-ray variability}

The $\gamma$-ray light curves are calculated using the unbinned likelihood analysis method implemented in the gtlike tool. The spectra of the considered sources were modeled by a power-law which can provide a good representation of the data over the small bins of time. The normalization and photon index of the sample sources are allowed to vary while the photon indexes of all background sources within the ROI are fixed to their best values obtained from the fit of the entire 10-year data set. As the diffuse background emission should not be variable, the parameters of the background models are fixed as well. During the fitting, the events within $0.1-300 \mathrm{GeV}$ with the appropriate quality cuts mentioned above are considered.

Initially, in order to study the variability, the $\gamma$-ray light curves with 30-day binning are calculated for all sources, considering the 0.1 $300 \mathrm{GeV}$ range. Additional light curves with short periods (days or a week) were computed for the statistically significant $(\geq 5 \sigma)$ $\gamma$-ray emitting periods identified in the monthly light curves. Next, using the adaptively binned method of Lott et al. (2012), the $\gamma$-ray variability is studied further. At fixed time binning, when long time intervals are used, a possible fast flux variation will be smoothed out, while using short bins might result in many upper limits during the low-activity periods. In the adaptive binning approach, the time bin widths are adjusted to produce bins with constant flux uncertainty above the optimal energy $\left(E_{0}\right)$ and this appraoch is proved to be a powerful method to search for $\gamma$-ray flux variation (e.g., Rani et al. 2013b; Sahakyan \& Gasparyan 2017; Sahakyan et al. 2018; Baghmanyan et al. 2017; Gasparyan et al. 2018; Dutka et al. 2017; Zargaryan et al. 2017). $E_{0}$ depends on the flux and photon index of each source and can be computed following Lott et al. (2012). For the considered sources, adaptive binning light curves with $15 \%$ uncertainty are generated.

\section{SWIFT OBSERVATIONS}

Swift is a multi-frequency space observatory, designed as a rapid response mission for follow-up observation of gamma-ray bursts (GRBs) (Gehrels et al. 2004). Although its primary scientific goal is the observation of GRBs, due to wide frequency coverage, it is suitable for blazar studies. The data from two of the instruments onboard Swift UVOT (170-600 nm; Roming et al. 2005) and XRT (0.3-10.0 keV; Burrows et al. 2005) have been analyzed in the current paper. Twenty-nine sources from Table 1 (except MRSS 291081526, CRATES J233930+024420, MG1 J154930+1708 and B3 $1624+414$ ) were at least once observed by Swift. There are available data from multiple observations of some sources; e.g., B2 0743+25, PKS 0438-43, TXS 0222+185, TXS 1448+093, PMN J0226+0937, TXS 1616+517 and PKS 2107-105 had been observed more than ten times. The Swift XRT data analysis was possible to apply only for twenty two sources (see Table 3) and twenty six were detected in at least one of the optical-UV Swift-UVOT filters (see Table 4). Swift UVOT data from all six bands are considered when available: UVW2 (188 nm), UVM2 (217 nm), UVW1 (251 nm), U (345 $\mathrm{nm}), \mathrm{B}(439 \mathrm{~nm})$ and $\mathrm{V}(544 \mathrm{~nm})$. The photometry analysis of all 


\begin{tabular}{|c|c|c|c|c|c|c|}
\hline Object & $\bar{V}$ & $\bar{B}$ & $\bar{U}$ & $\bar{W} W 1$ & $\overline{M 2}$ & $\bar{W}$ \\
\hline PKS 1351-108 - & - & - & $(4.54 \pm 2.53) \times 10^{-14}$ & - & - & \\
\hline MG3 J163554+3629 & - & - & $(6.93 \pm 2.72) \times 10^{-14}$ & - & - & $(7.50 \pm 4.11) \times 10^{-14}$ \\
\hline PKS 0537-286 & $(3.78 \pm 5.86) \times 10^{-15}$ & $(4.53 \pm 1.56) \times 10^{-14}$ & $(3.54 \pm 0.42) \times 10^{-13}$ & $(4.54 \pm 2.54) \times 10^{-14}$ & $(6.84 \pm 0.75) \times 10^{-14}$ & $(4.61 \pm 0.55) \times 10^{-13}$ \\
\hline TXS 0800+618 & $(7.69 \pm 0.12) \times 10^{-15}$ & - & $(2.39 \pm 0.6) \times 10^{-13}$ & $(5.68 \pm 0.18) \times 10^{-15}$ & - & $(6.58 \pm 0.13) \times 10^{-14}$ \\
\hline S4 $1427+543$ & - & $(2.52 \pm 2.03) \times 10^{-14}$ & $(2.53 \pm 0.92) \times 10^{-13}$ & - & $(3.88 \pm 1.4) \times 10^{-14}$ & $(1.50 \pm 0.02) \times 10^{-15}$ \\
\hline GB6 J0733+0456 & - & $(2.97 \pm 2.55) \times 10^{-14}$ & - & $(9.40 \pm 0.25) \times 10^{-15}$ & - & - \\
\hline B2 $0743+25$ & - & $(4.92 \pm 0.82) \times 10^{-14}$ & $(3.39 \pm 0.59) \times 10^{-13}$ & - & $(1.12 \pm 0.22) \times 10^{-13}$ & $(4.76 \pm 0.76) \times 10^{-13}$ \\
\hline PKS 0347-211 & - & - & $(2.80 \pm 0.52) \times 10^{-13}$ & - & $(1.69 \pm 0.5) \times 10^{-13}$ & $(2.03 \pm 1.09) \times 10^{-13}$ \\
\hline S4 1124+57 & $(1.16 \pm 0.85) \times 10^{-14}$ & - & - & - & - & - \\
\hline PKS 0438-43 & $(7.90 \pm 0.1) \times 10^{-15}$ & - & $(3.45 \pm 0.38) \times 10^{-13}$ & - & - & $(8.02 \pm 0.69) \times 10^{-13}$ \\
\hline S4 2015+65 & $(1.63 \pm 0.02) \times 10^{-15}$ & - & - & $(6.91 \pm 0.33) \times 10^{-14}$ & - & - \\
\hline 87 GB $214302.1+095227$ & $(1.01 \pm 0.48) \times 10^{-13}$ & $(2.49 \pm 0.36) \times 10^{-13}$ & - & $(2.78 \pm 0.72) \times 10^{-13}$ & $(3.49 \pm 0.63) \times 10^{-13}$ & - \\
\hline MG2 J174803+3403 & $(1.92 \pm 0.32) \times 10^{-13}$ & $(2.53 \pm 0.25) \times 10^{-13}$ & $(4.68 \pm 0.87) \times 10^{-13}$ & $(2.40 \pm 0.33) \times 10^{-13}$ & $(3.73 \pm 0.61) \times 10^{-13}$ & $(3.20 \pm 1.52) \times 10^{-13}$ \\
\hline PKS 0834-20 & - & - & $(7.82 \pm 0.54) \times 10^{-13}$ & $(3.84 \pm 1.72) \times 10^{-14}$ & $(2.39 \pm 0.36) \times 10^{-13}$ & $(7.29 \pm 0.91) \times 10^{-13}$ \\
\hline TXS $0222+185$ & - & - & $(9.36 \pm 0.89) \times 10^{-13}$ & - & $(8.18 \pm 1.11) \times 10^{-13}$ & $(1.12 \pm 0.21) \times 10^{-12}$ \\
\hline OD 166 & - & - & - & - & - & $(9.19 \pm 0.36) \times 10^{-14}$ \\
\hline TXS $0907+230$ & $(9.53 \pm 4.27) \times 10^{-14}$ & $(9.66 \pm 3.17) \times 10^{-14}$ & $(5.28 \pm 2.66) \times 10^{-13}$ & $(8.77 \pm 3.31) \times 10^{-14}$ & $(2.09 \pm 1.55) \times 10^{-13}$ & - \\
\hline PMN J1441-1523 & - & - & $(6.33 \pm 4.81) \times 10^{-14}$ & - & - & - \\
\hline CRATES J05433+392803 & - & - & - & $(1.90 \pm 0.88) \times 10^{-16}$ & - & - \\
\hline TXS 1448+093 & - & - & $(6.53 \pm 2.36) \times 10^{-14}$ & - & - & $(1.35 \pm 0.35) \times 10^{-13}$ \\
\hline PMN J0226+0937 & $(9.95 \pm 4.17) \times 10^{-14}$ & $(5.13 \pm 0.9) \times 10^{-13}$ & $(1.22 \pm 0.06) \times 10^{-12}$ & $(4.39 \pm 1.53) \times 10^{-13}$ & $(1.30 \pm 0.06) \times 10^{-12}$ & $(1.26 \pm 0.08) \times 10^{-12}$ \\
\hline PKS 0451-28 & - & $(9.52 \pm 1.21) \times 10^{-14}$ & - & - & - & - \\
\hline B3 0908+416B & $(3.45 \pm 0.11) \times 10^{-15}$ & $(3.46 \pm 2.22) \times 10^{-14}$ & $(2.08 \pm 0.66) \times 10^{-13}$ & $(4.94 \pm 1.95) \times 10^{-14}$ & $(6.87 \pm 1.91) \times 10^{-14}$ & $(2.67 \pm 1.2) \times 10^{-13}$ \\
\hline TXS $1616+517$ & - & - & $(1.96 \pm 0.27) \times 10^{-13}$ & - & - & $(1.25 \pm 0.34) \times 10^{-13}$ \\
\hline B3 1343+451 & - & $(3.12 \pm 2.77) \times 10^{-14}$ & $(4.54 \pm 0.55) \times 10^{-13}$ & $(7.41 \pm 0.23) \times 10^{-15}$ & $(2.52 \pm 0.29) \times 10^{-13}$ & $(4.47 \pm 0.65) \times 10^{-13}$ \\
\hline PKS 2107-105 & - & - & $(1.74 \pm 0.1) \times 10^{-12}$ & - & - & $(1.44 \pm 0.13) \times 10^{-12}$ \\
\hline
\end{tabular}


our sources was performed using the standard UVOT software distributed within the HEAsoft package (v6.25) and the calibration included in the CALDB (v.20170922). The source counts for each filter are extracted from a circular region with a 5 " radius around the sources, while the background ones from a region with $20^{\prime \prime}$ radius not being contaminated with any signal from the nearby sources. uvotsource tool was used to convert the source counts using the conversion factors provided by Poole et al. (2008). The fluxes were corrected for extinction using the reddening coefficient $E(B-V)$ from the Infrared Science Archive ${ }^{1}$. In the case of several observations for the same source, the analysis is performed using the same background region but validated that it is not contaminated by nearby objects in any filter. Also, light curves have been generated for investigation of the flux variation in each band. Then, if no significant variation is found, the spectral points are computed from the summed images, resulting in the flux estimation with reduced uncertainties.

The XRT data, taken both in photon counting and window timing modes were analyzed with standard XRTDAS tool distributed within the HEAsoft package (v6.25), applying standard procedures, filtering and screening criteria. The source counts were selected from a 20-pixel $\left(47^{\prime \prime}\right)$ circle centered on the coordinates of each source, while those for the background- from an annulus with the same center and inner and outer radii of $51\left(120^{\prime \prime}\right)$ and 85 pixels $\left(200^{\prime \prime}\right)$, respectively. The Cash statistics (Cash 1979) on ungrouped data was used, as for many observations the number of counts was low and did not contain the minimum number of counts required for Gaussian statistics. The $0.3-10 \mathrm{keV}$ X-ray spectrum of each source is fitted with XSPEC12.10.1 adopting an absorbed power-law model with a neutral hydrogen column density fixed to its Galactic value in each direction.

Initially, for the considered sources, the X-ray spectral analysis was performed for each observation. However, for most of the sources, the count rate was below 20, preventing spectral fitting, and the photon index and flux could be estimated only for a few bright sources. Then, when several observations of the same source were available, they were merged to increase the photon statistics, and the averaged $\mathrm{X}$-ray spectra were obtained. The merging was done using the tool available from the UK Swift Science Data Centre ${ }^{2}$ (Evans et al. 2009). Again XSPEC was used to fit the $0.3-10 \mathrm{keV}$ spectrum, testing both the absorbed power-law and log-parabola models. We note that the spectra of many sources could be constrained only by merging the observations.

\section{RESULTS OF DATA ANALYSES}

The $\gamma$-ray data analysis results obtained from the power-law fit in the range from $100 \mathrm{MeV}$ to $500 \mathrm{GeV}$ are presented in Table 1 for each source, providing the $\gamma$-ray flux $\left(F_{\gamma}\right)$, photon index, the detection significance (TS), the energy of the highestenergy events $\left(E_{\max }\right)$ detected from each object with the probability of its association with the target. The sources are detected with $T S>34.05$ significance, except for S4 2015+65, which appeared with $T S=23.77$. B3 $1343+451$ is detected with the highest significance of $T S=34652.79(186.15 \sigma)$, allowing to perform a detailed spectral and variability analysis. In addition, for the sources with the spectra modeled by a log-parabola in 4FGL, an additional fit with

\footnotetext{
${ }^{1}$ http://irsa.ipac.caltech.edu/applications/DUST/

2 https://www.swift.ac.uk/user_objects/
}

a log-parabola model was performed, the results of which are presented in Table 2. The curvature of the spectra of PKS 1351-018, B2 0743+25, PKS 0438-43, S4 2015+65, PKS 0834-20, and OD 166 is substantial $(\beta=0.24-0.63)$, so their $\gamma$-ray spectrum quickly declines. The flux estimated from the power-law fitting which yielded soft $\gamma$-ray spectra as well does not substantially differ from that obtained with a log-parabola. The only noticeable difference is found for PKS 1351-018; the flux estimated from the log-parabola fitting is $(6.86 \pm 1.66) \times 10^{-9}$ photon $\mathrm{cm}^{-2} \mathrm{~s}^{-1}$ as compared with $(1.11 \pm 0.15) \times 10^{-8}$ photon $\mathrm{cm}^{-2} \mathrm{~s}^{-1}$ when the spectrum was modeled by a power-law. However, for this source $\beta=0.63 \pm 0.23$ was estimated implying its spectrum is curved significantly. Interestingly, the log-parabola fitting of S4 2015+65 spectrum resulted in detection of the source with $T S=27.96$.

The results presented in Table 1 are shown in Fig. 1. The $\gamma$-ray photon index versus flux (estimated from power-law fitting) is shown in the upper left panel, where the FSRQs are circles, BL LACs triangles and BCUs squares. Even though the number of sources is not enough for population studies, some difference in various blazar types can be seen. The photon index $\left(\sim E^{-\Gamma_{\gamma}}\right)$ estimated in the $0.1-500 \mathrm{GeV}$ range ranges from 1.71 to 3.05 with a mean of 2.54 . The soft $\gamma$-ray spectra of the considered sources (except 87 GB 214302.1+095227 and MG1 J154930+1708) indicate that the peak of the HE component in their SED is in the $\mathrm{MeV}$ range. The flux of considered sources ranges from $4.84 \times 10^{-10}$ to $1.50 \times 10^{-7}$ photon $\mathrm{cm}^{-2} \mathrm{~s}^{-1}$. The two BL Lacs detected beyond $z=2.5,87$ GB 214302.1+095227 and MG1 J154930+1708, have the lowest flux, $(4.84 \pm 1.37) \times$ $10^{-10}$ photon $\mathrm{cm}^{-2} \mathrm{~s}^{-1}$ and $(1.66 \pm 0.79) \times 10^{-9}$ photon $\mathrm{cm}^{-2} \mathrm{~s}^{-1}$, respectively, but they have a harder $\gamma$-ray spectrum. The $\gamma$-ray photon index of $87 \mathrm{~GB} 214302.1+095227$, which is the only ISP object among the selected sources, is $1.71 \pm 0.19$ and that of MG1 $\mathrm{J} 154930+1708$ is $\Gamma_{\gamma}=2.01 \pm 0.16$. The $\gamma$-ray flux of BCUs included in the sample ranges from $2.19 \times 10^{-9}$ photon $\mathrm{cm}^{-2} \mathrm{~s}^{-1}$ to $1.46 \times 10^{-8}$ photon $\mathrm{cm}^{-2} \mathrm{~s}^{-1}$ with $\Gamma_{\gamma}$ within $2.20-2.84$. The FSRQs occupy the region of high flux $\geq 3.89 \times 10^{-9}$ photon $\mathrm{cm}^{-2} \mathrm{~s}^{-1}$ and $\Gamma_{\gamma} \geq 2.2$ with a mean $F_{\gamma} \simeq 2.11 \times 10^{-8}$ photon $\mathrm{cm}^{-2} \mathrm{~s}^{-1}$. The highest $\gamma$-ray flux of $(1.50 \pm 0.02) \times 10^{-7}$ photon $\mathrm{cm}^{-2} \mathrm{~s}^{-1}$ was observed from the bright FSRQ B3 1343+451.

The highest energy events $\left(E_{\max }\right)$ along with the probability of being associated with the sources are given in Table 1. As the sources mostly have a soft $\gamma$-ray spectrum or the $\gamma$-ray data are better modeled by a log-parabola, their $\gamma$-ray spectra do not extend to HEs and the photon energies are below $20 \mathrm{GeV}$; except for $87 \mathrm{~GB}$ 214302.1+095227, MG2 J174803+3403, PMN J0226+0937 and B3 $1343+451$ from which photons with $67.15,31.63,56.42$ and 24.25 $\mathrm{GeV}$ have been detected.

The flux, which is a function of the distance, is compared for each source in Fig. 1 (upper left panel). Even though it is informative, the total energy released from each source cannot be investigated. Next, using the observed flux $\left(F_{\gamma}\right)$ and photon index $\left(\Gamma_{\gamma}\right)$, the luminosity of each source is computed as:

$L_{\gamma}=4 \pi d_{\mathrm{L}}^{2} \frac{\Gamma_{\gamma}-1}{\Gamma_{\gamma}-2} \frac{\mathrm{E}_{\max }^{2-\Gamma_{\gamma}}-\mathrm{E}_{\min }^{2-\Gamma_{\gamma}}}{\mathrm{E}_{\max }^{1-\Gamma_{\gamma}}-\mathrm{E}_{\min }^{1-\Gamma_{\gamma}}} F_{\gamma}$

where $\mathrm{E}_{\min }=100 \mathrm{MeV}$ and $\mathrm{E}_{\max }=500 \mathrm{GeV}$. Fig. 1 (upper right panel) shows the distribution of the considered sources in the $\Gamma_{\gamma}-L_{\gamma}$ plane. The $\gamma$-ray luminosity of considered sources ranges from $1.01 \times 10^{47} \mathrm{erg} \mathrm{s}^{-1}$ to $5.54 \times 10^{48} \mathrm{erg} \mathrm{s}^{-1}$. The lowest luminosity of $(1.01 \pm 0.38) \times 10^{47} \mathrm{erg} \mathrm{s}^{-1}$ has been estimated for CRATES $\mathrm{J} 105433+392803$ which is of the same order with that of the two BL Lacs included in the sample; $(1.42 \pm 0.98) \times 10^{47} \mathrm{erg} \mathrm{s}^{-1}$ 

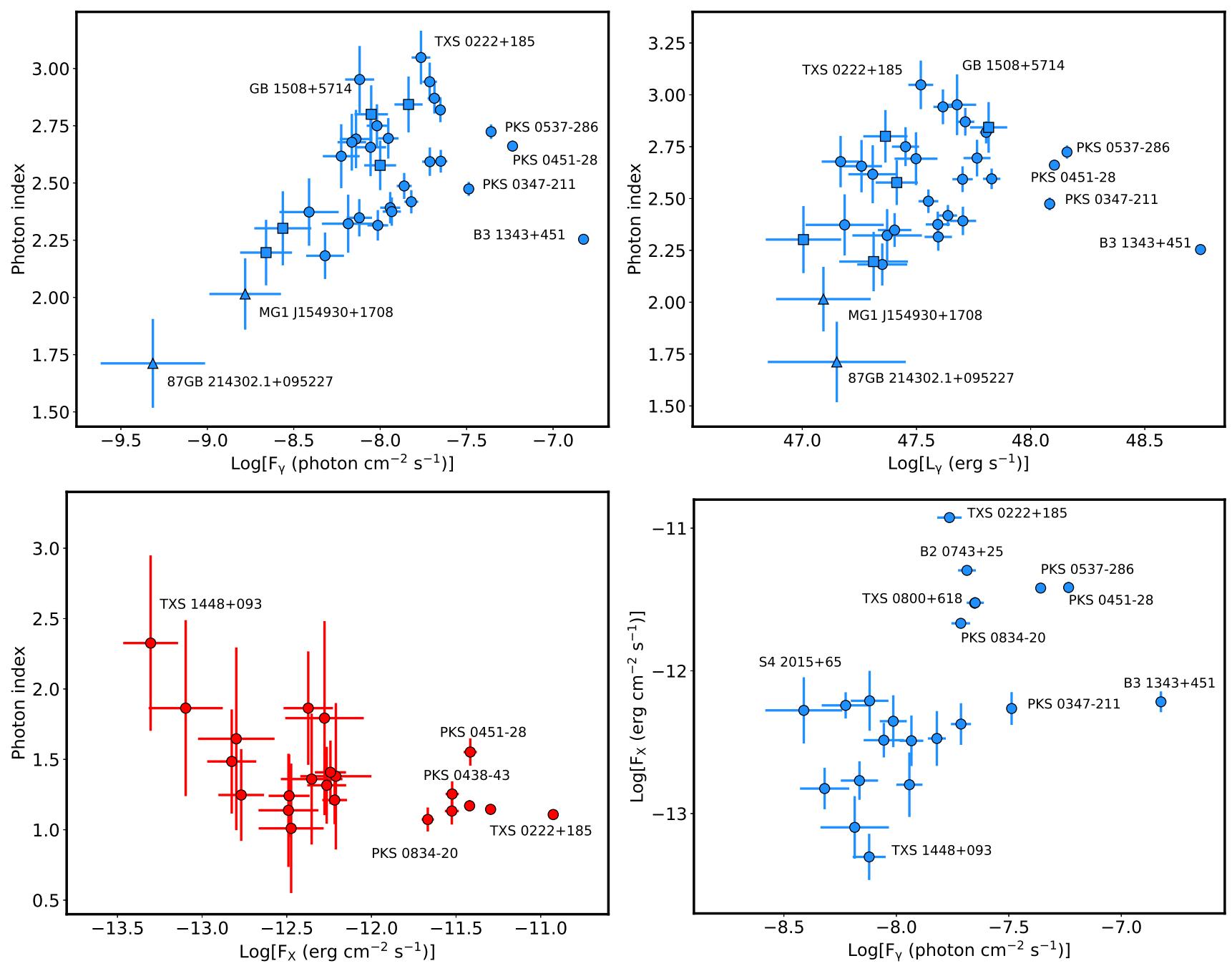

Figure 1. The $\gamma$-ray flux ( $>100 \mathrm{MeV}$ ) and luminosity of considered sources versus the photon index are shown in the upper panels. BL lacs, FSRQs and BCUs are shown with triangles, circles, and squares, respectively. Lower left panel: The X-ray flux $(0.3-10 \mathrm{keV})$ versus the photon index. Lower right panel: Comparison of $\gamma$-ray and X-ray (if available) fluxes.

for $87 \mathrm{~GB} 214302.1+095227$ and $(1.24 \pm 0.59) \times 10^{47} \mathrm{erg} \mathrm{s}^{-1}$ for MG1 J154930+1708. The luminosity of these BL Lacs corresponds to the highest end of the luminosity distribution of BL Lacs included in the fourth catalog of AGNs detected by Fermi-LAT (Fig. 10 in Ajello et al. 2020). The $\gamma$-ray luminosity of only PKS 0347-211, PKS 0451-28, PKS 0537-286 and B3 $1343+451$ exceeds $10^{48} \mathrm{erg} \mathrm{s}^{-1}$ with the highest $\gamma$-ray luminosity of $(5.54 \pm 0.06) \times 10^{48} \mathrm{erg} \mathrm{s}^{-1}$, estimated for B3 $1343+451$. Naturally, as compared to the distribution of all $\gamma$-ray emitting BL Lacs and FSRQs in the $\Gamma_{\gamma}-L_{\gamma}$ plane (Ajello et al. 2020), the blazars considered here occupy the highest luminosity range. We note that the luminosities shown in Fig. 1 (upper right panel) have been computed based on the time-averaged $\gamma$-ray flux, and even higher luminosities are expected during $\gamma$-ray flares.

Table 3 shows the X-ray data analysis results, for each source presenting the neutral hydrogen column density, X-ray photon index $\left(\Gamma_{X}\right)$, flux, and $\mathrm{C}$-stat/dof. The flux ranges from $\simeq 5 \times$ $10^{-14} \mathrm{erg} \mathrm{cm}^{-2} \mathrm{~s}^{-1}$ to $\simeq 10^{-11} \mathrm{erg} \mathrm{cm}^{-2} \mathrm{~s}^{-1}$, the highest flux of $(1.19 \pm 0.04) \times 10^{-11} \mathrm{erg} \mathrm{cm}^{-2} \mathrm{~s}^{-1}$ being observed for TXS $0222+185(z=2.69)$. Interestingly, from the sources considered here, only FSRQs are detected in the X-ray band; among BL Lacs, there are no observations for MG1 J154930+1708, while for 87GB $214302.1+095227$, even after merging six observations, only 10 counts are detected. Also, BCUs included in Table 1 have not been detected in the X-ray band.

The X-ray flux is plotted versus the photon index in the lower left panel of Fig. 1. For several sources, the number of detected counts was not high enough, so the flux and photon index were estimated with large uncertainties. The X-ray photon index of considered sources is $<2.0$, implying the $\mathrm{X}$-ray spectra have a rising shape in the $v F v \sim v^{2-\Gamma_{\mathrm{X}}}$ representation, which is natural as LSP blazars are considered. The only exception is TXS 1448+093 with $\Gamma_{\mathrm{X}}=2.33 \pm 0.62$, but even when merging its all 26 observations, the observed counts were only 25 . This source is relatively faint in the X-ray band with a flux of $(5.01 \pm 1.85) \times 10^{-14} \mathrm{erg} \mathrm{cm}^{-2} \mathrm{~s}^{-1}$, so even an exposure of $2.39 \times 10^{4} \mathrm{sec}$ is not enough to detect a reasonable number of counts. B3 0908+416B has the hardest X-ray spectrum with $\Gamma_{X}=1.01 \pm 0.46$. In the $F_{X}-\Gamma_{X}$ plane, PKS 0451-28, TXS 0222+185, PKS 0834-20, PKS 0537-286, TXS $0800+618$, B2 $0743+25$ and PKS $0438-43$ are detached from the other sources because they have a comparably high X-ray flux, $F_{\mathrm{X} \text {-ray }} \geq 2.13 \times 10^{-12} \mathrm{erg} \mathrm{cm}^{-2} \mathrm{~s}^{-1}$. In the lower right panel of 
Fig. 1, the $\gamma$-ray and X-ray (if available) fluxes of the considered sources are compared. Interestingly, the bright $\gamma$-ray sources PKS 0537-286 and PKS 0451-28 appear to be also bright X-ray emitters. The other bright $\gamma$-ray blazars (e.g., B3 1343+451, PKS 0451-28) do not have any distinguishable feature in the X-ray band, having a flux and photon index similar to those of the other considered sources. The bright X-ray sources TXS $0222+185$, B2 $0743+25$, TXS 0800+618, and PKS 0834-20 appear with a similar flux in the $\gamma$-ray band, $F_{\gamma}=(1.72-2.23) \times 10^{-8}$ photon $\mathrm{cm}^{-2} \mathrm{~s}^{-1}$. In Fig. 6 the X-ray spectra of all sources included in Table 3 are shown in red.

The Swift UVOT data analysis was performed in all available filters. The results are consistent when different background regions are selected. Initially, all the single observations of the sources were analyzed to search for variability. However, the sources are relatively faint in the optical/UV bands and the large uncertainties in the flux estimation do not allow to investigate flux variation in time. Table 4 summarizes the results of the UVOT data analysis after merging the observations for each source, presenting the fluxes in the six UVOT filters (if available) with errors. In Fig. 6 these data are shown in light blue. In the SED of GB 1508+5714, PKS 1351-108, PKS 0537-286, TXS 0800+618, S4 1427+543, GB6 J0733+0456, B2 0743+25, PKS 0347-211, S41124+57, PKS 0438-43, S4 2015+65, PKS 0834-20, TXS 0222+185, TXS 0907+230, PMN J0226+0937, PKS 0451-28 and PKS 2107-105, a thermal blue-bump component can be seen, which may represent the emission directly from the disc (Ghisellini et al. 2009b).

\subsection{X-ray variability}

The X-ray flux variability of some sources observed by Swift multiple times have been investigated. The data from each observation were processed and analyzed, but only for eight objects (marked with asterisks in Table 3) the number of counts was enough to constrain the flux and the photon index in a single observation. Except for PKS 0438-43, B2 $0743+25$ and TXS 0222+185, the $\mathrm{X}$-ray emission appeared to be relatively constant, though the sources were observed in different years. For example, the X-ray flux of PKS 0537-286 was $(4.18 \pm 0.74) \times 10^{-12} \mathrm{erg} \mathrm{cm}^{-2} \mathrm{~s}^{-1}$ and $(4.53 \pm 0.89) \times 10^{-12} \mathrm{erg} \mathrm{cm}^{-2} \mathrm{~s}^{-1}$ on 26 October, 2006 and 12 May 2017, respectively. Fitting the flux observed in different years with a constant flux, the $\chi^{2}$ test results in $P\left(\chi^{2}\right)=0.38$ and $\chi 2 / d o f=1.07$ which are consistent with no variability. Similarly, the $\chi 2$ test shows that the X-ray flux of TXS $0800+618$, PKS 0834-20, PKS 0451-28 and B3 1343+451 is constant over different years. There is a marginal evidence $\left(P\left(\chi^{2}\right)=0.038\right.$ and $\chi 2 /$ dof $=1.68)$ that the X-ray flux of TXS $0222+185$ is variable; it is mostly around $(0.96-1.1) \times 10^{-11} \mathrm{erg} \mathrm{cm}^{-2} \mathrm{~s}^{-1}$ which increased to $(1.62 \pm 0.13) \times 10^{-11} \mathrm{erg} \mathrm{cm}^{-2} \mathrm{~s}^{-1}$ and $(1.37 \pm 0.16) \times$ $10^{-11} \mathrm{erg} \mathrm{cm}^{-2} \mathrm{~s}^{-1}$ on 24 December 2014 and 31 July 2006, respectively. Instead, the $\chi 2$ test shows that the X-ray emission of PKS 0438-43 and B2 $0743+25$ is variable with $P(\chi 2)<5.1 \times 10^{-6}$. PKS 0438-43 was in a bright X-ray state on 15 December 2016 with a flux of $(1.09 \pm 0.16) \times 10^{-11} \mathrm{erg} \mathrm{cm}^{-2} \mathrm{~s}^{-1}$ as compared with the flux of $(1.30 \pm 0.31) \times 10^{-11} \mathrm{erg} \mathrm{cm}^{-2} \mathrm{~s}^{-1}$ in the quiescent state. Similarly, the X-ray flux of B2 $0743+25$ in the high state on 01 January 2006 was $(1.06 \pm 0.56) \times 10^{-11} \mathrm{erg} \mathrm{cm}^{-2} \mathrm{~s}^{-1}$. The variation of the 0.3-10 keV X-ray flux of PKS 0438-43, B2 0743+25 and TXS $0222+185$ is shown in Fig. 4. No variation of X-ray photon index was found, due to the large uncertainties in its estimation.

\section{$5.2 \gamma$-ray variability}

The continuous observation of the considered sources by FermiLAT allows a detailed investigation of their $\gamma$-ray flux variation during the considered ten years. When the source detection significance is $<10 \sigma$, the data are not enough for variability searches in month scales. Their emission could be variable in longer scales (6 or 12 months) more common for non-blazar AGNs (Sahakyan et al. 2018), which is not investigated here. Initially, the light curves of all sources were calculated with the help of an adaptive binning method. However, the adaptively binned intervals were possible to compute only for the source with a detection significance of $>37 \sigma$. The adaptively binned light curves of B3 1343+451, PKS 0451-28, PKS 0347-211 and PKS 0537-286 computed above $E_{0}=163.9,163.2,187.4$ and $151.0 \mathrm{MeV}$, respectively, are shown in Fig. 2. B3 1343+451 is the brightest and most variable source in the sample, and in its adaptively binned light curve (Fig. 2 a) several bright $\gamma$-ray emission periods can be identified: around MJD 55100, MJD 55890, MJD 56170, MJD 56640 and MJD 57060. During the bright $\gamma$-ray flaring periods, the flux changes in sub-day scales; there are 200 adaptive bins with a high flux and a width shorter than a day; the minimum adaptively binned time width is 6.33 hours observed on MJD 56176.34 when the source flux was $(7.52 \pm 1.85) \times 10^{-7}$ photon $\mathrm{cm}^{-2} \mathrm{~s}^{-1}$ (above $163.9 \mathrm{MeV}$ ). Moreover, the width of 83 bins from these 200 is even shorter than 12 hours, which were mostly observed during the flares around MJD 55890 (24 bins) and MJD 56170 (35 bins). In the quiescent state (e.g., before MJD 55800, expect for the flare on MJD 55100) the $\gamma$-ray flux of the source is $\simeq(1-5) \times 10^{-8}$ photon $\mathrm{cm}^{-2} \mathrm{~s}^{-1}$ which is lower than that averaged over 10 years (including the bright flaring states), reported in Table 1 . The highest $\gamma$-ray flux of $(8.77 \pm 2.16) \times 10^{-7}$ photon $\mathrm{cm}^{-2} \mathrm{~s}^{-1}$ above $E_{0}=163.9 \mathrm{MeV}$ with $\Gamma_{\gamma}=2.48 \pm 0.29$ was observed on MJD 55891.7 with a detection significance of $9.1 \sigma$ within a time bin having a width of 8.2 hours. It corresponds to a flux of $(1.82 \pm 0.45) \times 10^{-6}$ photon $\mathrm{cm}^{-2} \mathrm{~s}^{-1}$ above $100 \mathrm{MeV}$ which is 36.4 times higher than the $\gamma$-ray flux of the source in the quiescent state (before MJD 55800) but the $\Gamma_{\gamma}$ is within the uncertainties of the value given in Table 1. During the hardest $\gamma$-ray emission period, $\Gamma_{\gamma}=1.45 \pm 0.21$ was detected on MJD 56432 with a significance of $7.2 \sigma$, which is unusual for this source. Interestingly, there are twelve periods, mostly during the $\gamma$-ray flares, when its $\gamma$-ray spectrum was hard $(\leq 1.70)$; one such period had been observed on MJD 55900.53 when, within a time bin having a width of 12.90 hours, $\Gamma_{\gamma}$ was $1.64 \pm 0.16$ with a detection significance of $10.79 \sigma$. In the quiescent state, its $\gamma$-ray luminosity is $(2-4) \times 10^{48} \mathrm{erg} \mathrm{s}^{-1}$ which increased up to $\sim 1.5 \times 10^{50} \mathrm{erg} \mathrm{s}^{-1}$ during the bright $\gamma$-ray flares (Fig. 2 (a)).

The most distant source showing a substantial $\gamma$-ray flux increase in short periods is PKS 0537-286 at $z=3.10$ (Fig. 2 b). The flaring activity of this source was first reported in (Cheung 2017) and the rapid (6 and 12 hours) $\gamma$-ray flux variations in Li et al. (2018). During the extreme $\gamma$-ray flaring period from MJD 57878.05 to MJD 57881.55 , the adaptively binned light curve confirms the intra-day $\gamma$-ray flux increase of PKS 0537-286; the adaptive time bin widths are $20.45,15.96$ and 18.90 hours. For comparison, during the flux increase around MJD 55540 and MJD 56390 the minimum time widths are 2.38 and 3.79 days, respectively. The average $\gamma$-ray flux of PKS 0537-286 is $(4.38 \pm 0.18) \times 10^{-8}$ photon $\mathrm{cm}^{-2} \mathrm{~s}^{-1}$ (Table 1), but it significantly increased in MJD 55528-55553, MJD 56264-56400 and MJD 57878.0-57883.4. In the last period, during five consecutive time intervals, the $\gamma$-ray flux above $151.0 \mathrm{MeV}$ was higher than $3 \times 10^{-7}$ photon $\mathrm{cm}^{-2} \mathrm{~s}^{-1}$, with a maximum of 


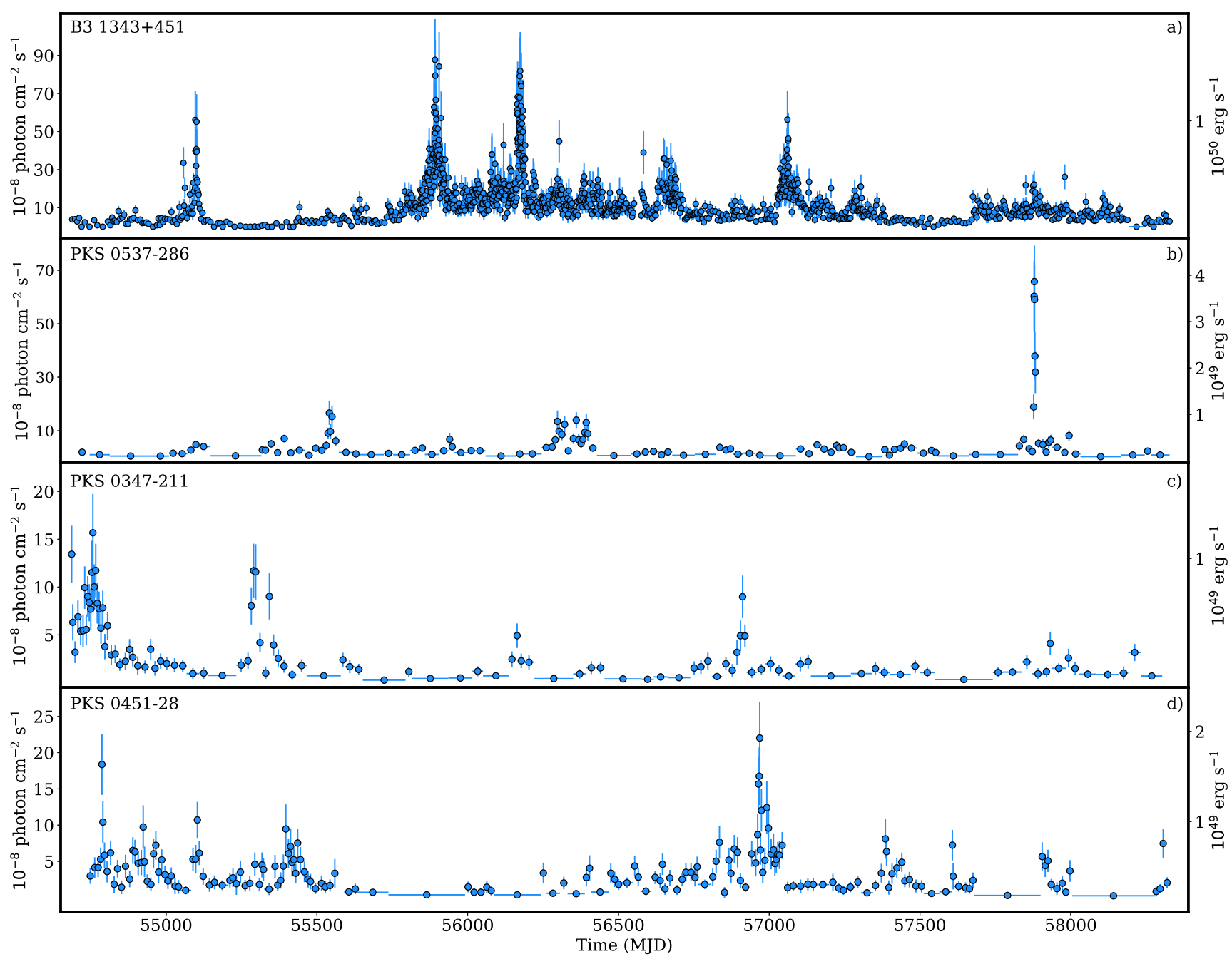

Figure 2. $\gamma$-ray light curve of B3 1343+451, PKS 0537-286, PKS 0347-211, and PKS 0451-28 for the period from August 2008 to August 2018 calculated using adaptively-binned timescales.

$(6.58 \pm 1.35) \times 10^{-7}$ photon $\mathrm{cm}^{-2} \mathrm{~s}^{-1}$ observed on MJD 57879.2 with a detection significance of $10.57 \sigma$. This corresponds to a flux of $(1.29 \pm 0.26) \times 10^{-6}$ photon $\mathrm{cm}^{-2} \mathrm{~s}^{-1}$ above $100 \mathrm{MeV}$. During these periods, $\Gamma_{\gamma}$ is $2.45 \pm 0.23,2.64 \pm 0.27,2.56 \pm 0.28,2.79 \pm 0.34$ and $2.91 \pm 0.35$, not significantly different from the value reported in Table 1 with no spectral hardening, which shows the emission is dominated by the $\mathrm{MeV}$ photons. However, during the flares, the luminosity of the source can be as high as $\simeq 4 \times 10^{49} \mathrm{erg} \mathrm{s}^{-1}$, putting PKS 0537-286 in the list of the brightest $\gamma$-ray blazars.

The adaptively binned light curves of PKS 0347-211 and PKS 0451-28 (Fig. 2 c and d) show several periods of $\gamma$-ray brightening, when a $\gamma$-ray flux increase within day scales is observed. For example, the shortest time interval when the flux increases is 2.65 days for PKS 0347-211, and it is 1.56 days for PKS 045128 . The light curves of both sources reveal several $\gamma$-ray flaring periods when the flux substantially increased. For example, on MJD 54757.04 \pm 2.71 the $\gamma$-ray flux of PKS 0347-211 above $187.4 \mathrm{MeV}$ was $(1.57 \pm 0.41) \times 10^{-7}$ photon $\mathrm{cm}^{-2} \mathrm{~s}^{-1}$, which corresponds to a flux of $(3.57 \pm 0.93) \times 10^{-7}$ photon $\mathrm{cm}^{-2} \mathrm{~s}^{-1}$ above $100 \mathrm{MeV}$. In the case of PKS 0451-28, the peak $\gamma$-ray flux of $(2.20 \pm 0.50) \times 10^{-7}$ photon $\mathrm{cm}^{-2} \mathrm{~s}^{-1}$ (above $163.2 \mathrm{MeV}$ ) was observed on MJD $56968.60 \pm 0.79$ with $9.64 \sigma$. This corresponds to a flux of $(3.70 \pm 0.84) \times 10^{-7}$ photon $\mathrm{cm}^{-2} \mathrm{~s}^{-1}$ above $100 \mathrm{MeV}$. During this period, $\Gamma_{\gamma}$ was $2.06 \pm 0.19$.

The light curves generated with the help of the adaptive binning method allowed to identify periods when the flux of some of the sources considered here (Fig. 2) increased in sub-day or days scales. It should be mentioned that expect for B3 1343+451, the short time scale variability of the other sources cannot be investigated using the regular time binning method, because in a large number of periods only upper limits are derived. Also, because of low statistics, the adaptively binned light curves were possible to compute only for the source presented in Fig. 2. For the other sources included in Table 1 the variability on week and month scales are investigated. In order to identify whether the $\gamma$-ray emission is variable or not, a simple $\chi 2$ test was performed (Abdo et al. 2010b); the flux measured in each interval was fitted by a constant flux and the reduced $\chi^{2}$ and the probability of the flux being constant are computed.

The $\chi 2$ fitting indicated that the $\gamma$-ray emission of B3 $0908+416 \mathrm{~B}$, TXS $0800+618$, PKS $0438-43$, OD 166 and TXS $0907+230$ is variability in week scales while that of MG3 J163554+3629, GB6 J0733+0456, B2 0743+25, PMN J1441-1523 and TXS 1616+517 in month scales. For all these sources, $P(\chi 2)<2.16 \times 10^{-4}$ was estimated. The $\gamma$-ray light curves of these sources with an 

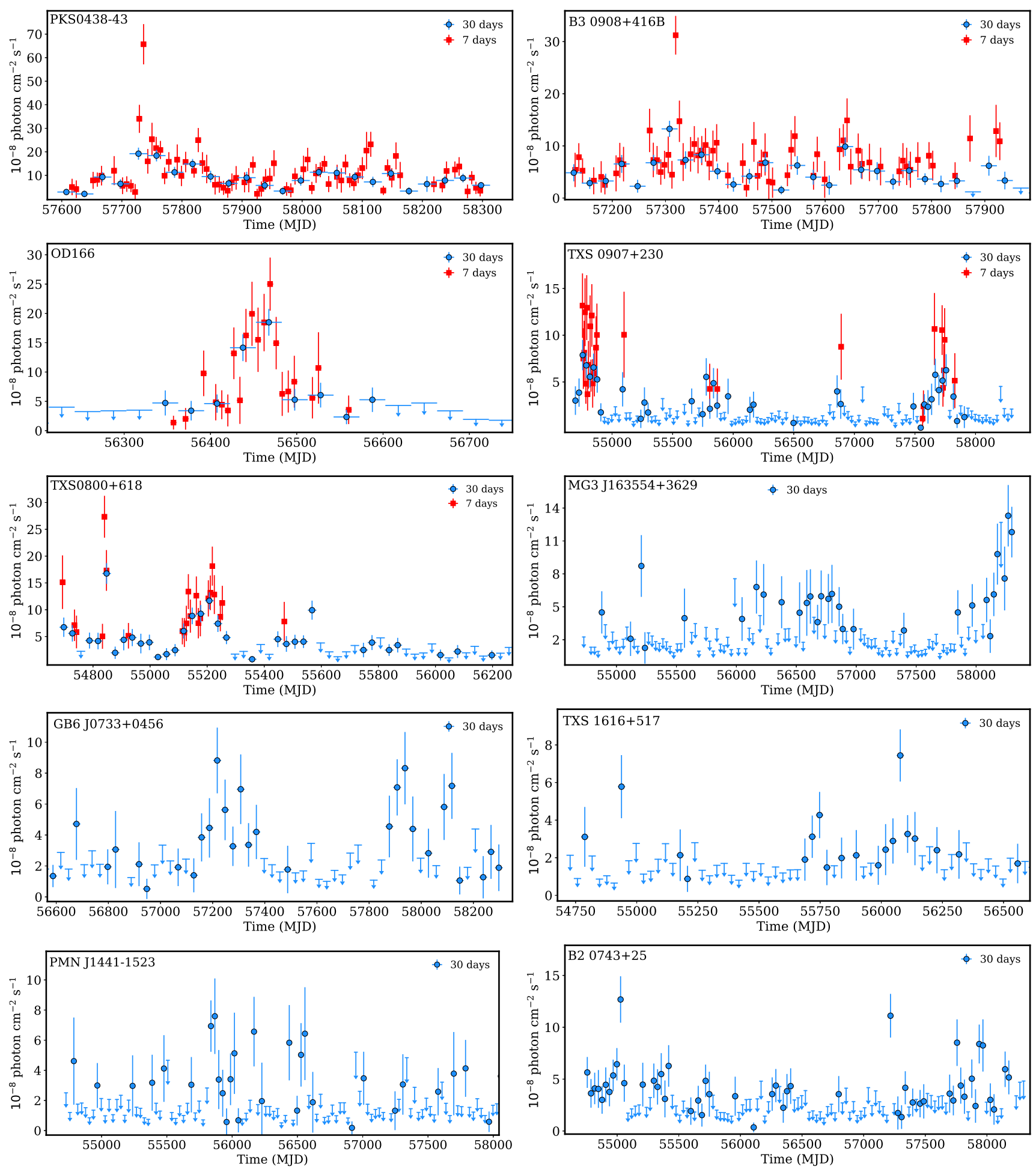

Figure 3. 7-day (red) and 30-day (blue) binned light light curves of PKS 0438-43, B3 0908+416B, OD 166, TXS 0907+230, TXS 0800+618, MG3 J163554+3629, GB6 J0733+0456, TXS 1616+517, PMNJ 1441-1523 and B2 0743+25.

evident increase in the flux are shown in Fig. 3. For example, during MJD $57729.16 \pm 3.5$ and MJD $57736.16 \pm 3.5$, the $\gamma$ ray flux of PKS 0438-43 above $100 \mathrm{MeV}$ increased $15.2-29.3$ times as compared to its average flux and was $(3.40 \pm 0.59) \times$ $10^{-7}$ photon $\mathrm{cm}^{-2} \mathrm{~s}^{-1}$ and $(6.57 \pm 0.86) \times 10^{-7}$ photon $\mathrm{cm}^{-2} \mathrm{~s}^{-1}$, respectively. Likewise, the 7 -day averaged peak values of the $\gamma$ - ray flux of B3 0908+416B, OD 166, TXS 0800+618 and TXS $0907+230$ were $(3.12 \pm 0.37) \times 10^{-7}$ photon $\mathrm{cm}^{-2} \mathrm{~s}^{-1},(2.50 \pm$ $0.45) \times 10^{-7}$ photon $\mathrm{cm}^{-2} \mathrm{~s}^{-1},(2.73 \pm 0.39) \times 10^{-7}$ photon $\mathrm{cm}^{-2} \mathrm{~s}^{-1}$ and $(1.32 \pm 0.34) \times 10^{-7}$ photon $\mathrm{cm}^{-2} \mathrm{~s}^{-1}$, respectively, which exceed the corresponding values given in Table 1 . The most distant $\gamma$-ray flaring blazar observed so far is MG3 J163554+3629 


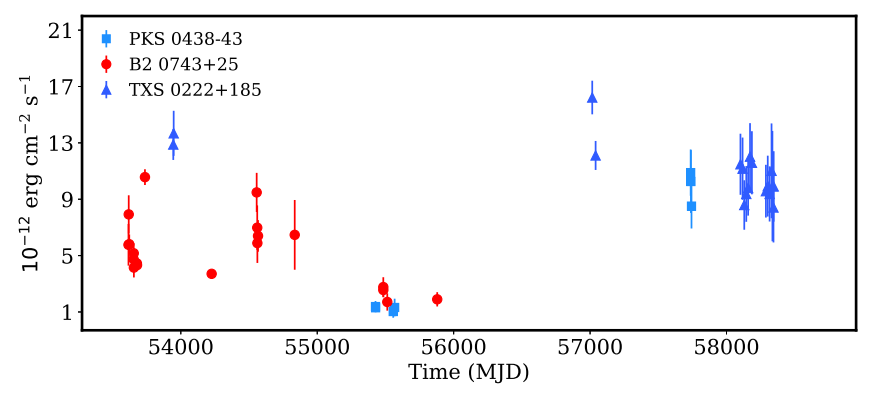

Figure 4. The 0.3-10 keV X-ray fluxes of PKS 0438-43, B2 0743+25 and TXS $0222+185$ measured by Swift-XRT.

at $z=3.65$; this source was reported to be in an active state on July 72018 , when its daily averaged peak value of $\gamma$-ray flux was $(6.4 \pm 1.15) \times 10^{-7}$ photon $\mathrm{cm}^{-2} \mathrm{~s}^{-1}$ (Paliya et al. 2018). The monthly averaged maximum $\gamma$-ray flux of $(1.33 \pm 0.28) \times$ $10^{-7}$ photon $\mathrm{cm}^{-2} \mathrm{~s}^{-1}$ was observed on MJD 58267.66 \pm 15 (MayJune 2018). The source was also bright in $\gamma$-rays with a flux of $(1.18 \pm 0.23) \times 10^{-7}$ photon $\mathrm{cm}^{-2} \mathrm{~s}^{-1}$ during June-July 2018 when the maximum daily averaged flux was observed (Paliya et al. 2018). For the considered sources, the $\gamma$-ray photon index evolution in time has also been investigated.The photon index is defined by the processes responsible for particle acceleration and cooling, and its significant changes are directly linked with the processes inside the jet. As the adaptively binned light curves have narrow time bins, the photon indexes are estimated with large uncertainties which introduces difficulties for investigation of their variability. Therefore, the $\gamma$-ray light curves produced in 30 and 7 days (when available) are used to search for photon index variation, except for B3 1343+451 for which a 3-day binned light curve has been used. Moreover, in the light curves only the periods when the source detection was $T S>25$ were considered, otherwise the large uncertainties on the photon index estimation would not allow to make definite conclusions. The simple $\chi 2$ test shows that among the considered sources only the photon index of B3 1343+451, PKS 0451-28, B3 0908+416B and TXS $0907+230$ varies in time: the variation is highly significant for B3 1343+451 and PKS 0451-28 with $P(\chi 2)=6.9 \times 10^{-4}$ and $P(\chi 2) \leq 10^{-5}$, respectively, and $P(\chi 2) \simeq 0.015$ for B $30908+416 \mathrm{~B}$ and TXS $0907+230$. The evolution of the photon index of these sources in time is shown in Fig. 5 were the horizontal line corresponds to the averaged photon index estimated in ten years (Table 1). The photon index of B3 1343+451 clearly varies, the hardest spectrum being observed on MJD 58089.16 \pm 1.5 with $\Gamma_{\gamma}=1.73 \pm 0.24$; in total there are 60 periods when $\Gamma_{\gamma}<2.0$. Interestingly, the $\gamma$-ray spectrum of the source was hard with $1.95 \pm 0.07$ when it was in a bright $\gamma$-ray flaring state on MJD 56172.16 \pm 1.5 . The $\gamma$-ray spectrum of PKS 0451-28 is usually soft but in the 30-day binned light curve the periods when $\Gamma_{\gamma}=2.06 \pm 0.07$ and $\Gamma_{\gamma}=2.17 \pm 0.15$ are observed on MJD 56977.66 \pm 15 and $58297.66 \pm 15$, respectively. The first period overlaps with the large $\gamma$-ray flare evident in the adaptively binned light curve (see Fig. 2 a). The hardening of the $\gamma$-ray spectrum of B3 0908+416B on MJD 57517.66 \pm 15 is remarkable when the $\Gamma_{\gamma}$ changed to $1.84 \pm 0.25$ compared to $2.42 \pm 0.05$ averaged over 10 years. TXS $0907+230$ is the most distant object in our sample $(z=2.66)$ with occasional hardening of its $\gamma$-ray spectrum. The 7-day binned light curve of TXS $0907+230$ shows that there are three periods (on MJD 54798.16 $\pm 3.5,54805.16 \pm 3.5$ and $54826.16 \pm 3.5$ ) when its $\gamma$-ray emission appears with an unusually hard $\gamma$-ray spectrum with $\Gamma_{\gamma}=1.72 \pm 0.23,1.90 \pm 0.21$ and
$1.72 \pm 0.15$. Yet, in the monthly binned light curve, in the bin covering these periods, $\Gamma_{\gamma}$ is $1.88 \pm 0.10$ with a detection significance of $12.5 \sigma$. Even if the $\gamma$-ray photon index of MG3 J163554+3629 and PMN J0226+0937 appeared to be constant, hardening of their $\gamma$-ray spectra was occasionally observed. For example, for MG3 $\mathrm{J} 163554+3629 \Gamma_{\gamma}=2.29 \pm 0.13$ (with $11.1 \sigma$ ) was observed from MJD 58282 to 58312, likewise for PMN J0226+0937 $\Gamma_{\gamma}$ was $1.80 \pm 0.16$ (with $9.7 \sigma$ ) on MJD 54892-54922. The hard $\gamma$-ray spectra of the sources discussed above are shown in Fig. 6 in magenta; substantial changes in the $\gamma$-ray spectrum are evident. Such a hard $\gamma$-ray spectrum is more typical for BL Lacs, but it has also been occasionally observed for the FSRQs during the flares (e.g., see Pacciani et al. 2014; Sahakyan \& Gasparyan 2017; Gasparyan et al. 2018; Sahakyan 2020; Paliya et al. 2019; Angioni et al. 2019).

In addition, $\gamma$-ray light curves with 30-day binning above $1 \mathrm{GeV}$ are produced to investigate the flux and photon index variation in the $\mathrm{GeV}$ band. The periods when the source emission is significant above $1 \mathrm{GeV}$ are also relevant for studying absorption through interaction with EBL photons. There are only a few periods when the considered sources have been detected above $1 \mathrm{GeV}$ with a sufficient significance $(>5 \sigma)$. The emission, in these periods, is mostly characterized by a soft $\gamma$-ray spectrum, implying these are the same components as those at lower energies. However, the periods when the sources were detected by Fermi-LAT correspond to only a small fraction of the total bins (30-day binning), so the poor statistics did not allow us to investigate the possible flux variation or photon index hardening above $1 \mathrm{GeV}$.

\section{THE ORIGIN OF MULTIWAVELENGTH EMISSION}

The multiwavelength emission from blazars produced when the accelerated electrons (Ghisellini et al. 1985; Bloom \& Marscher 1996; Maraschi et al. 1992; Błażejowski et al. 2000; Sikora et al. 1994; Ghisellini \& Tavecchio 2009) or protons (Dar \& Laor 1997; Beall \& Bednarek 1999; Bednarek \& Protheroe 1997; Mannheim 1995; Mannheim \& Biermann 1989; Mannheim 1993; Mücke \& Protheroe 2001; Mücke et al. 2003) interact with the magnetic and photon fields, contain valuable information necessary for understanding the physics of their jets. The currently proposed models can explain a single SED snapshot, but they cannot selfconsistently explain the dynamical evolution of the radiation and origin of the flares. The flares are most likely caused by changes in the radiating particles or in the emission region (e.g., Paggi et al. 2011), and their origin can be investigated only with contemporaneous multiwavelength data. During the $\gamma$-ray flaring of the considered sources (Figs. 2 and 3), contemporaneous Swift data are available only for PKS 0438-43 and TXS 0800+618. However, the Swift observations in these periods show that the $\mathrm{X}$-ray photon index and flux did not change, being the same as those given in Table 3, whereas in the optical/UV bands these sources were detected only in one or two UVOT filters with large uncertainties in the flux estimation. So, it is not clear whether or not the low energy component changed or remained the same during the $\gamma$-ray flares. This introduces uncertainties for the theoretical modeling and does not allow to model the SEDs of these two objects during their $\gamma$-ray flaring. Moreover, as there are no multiwavelength data during the $\gamma$-ray flares of the other sources and our goal is to constrain the main physical parameters of distant blazar jets, only the averaged multiwavelength SEDs have been used in the modeling which represent the typical state of the sources.

To understand the origin of the broadband emission of the con- 

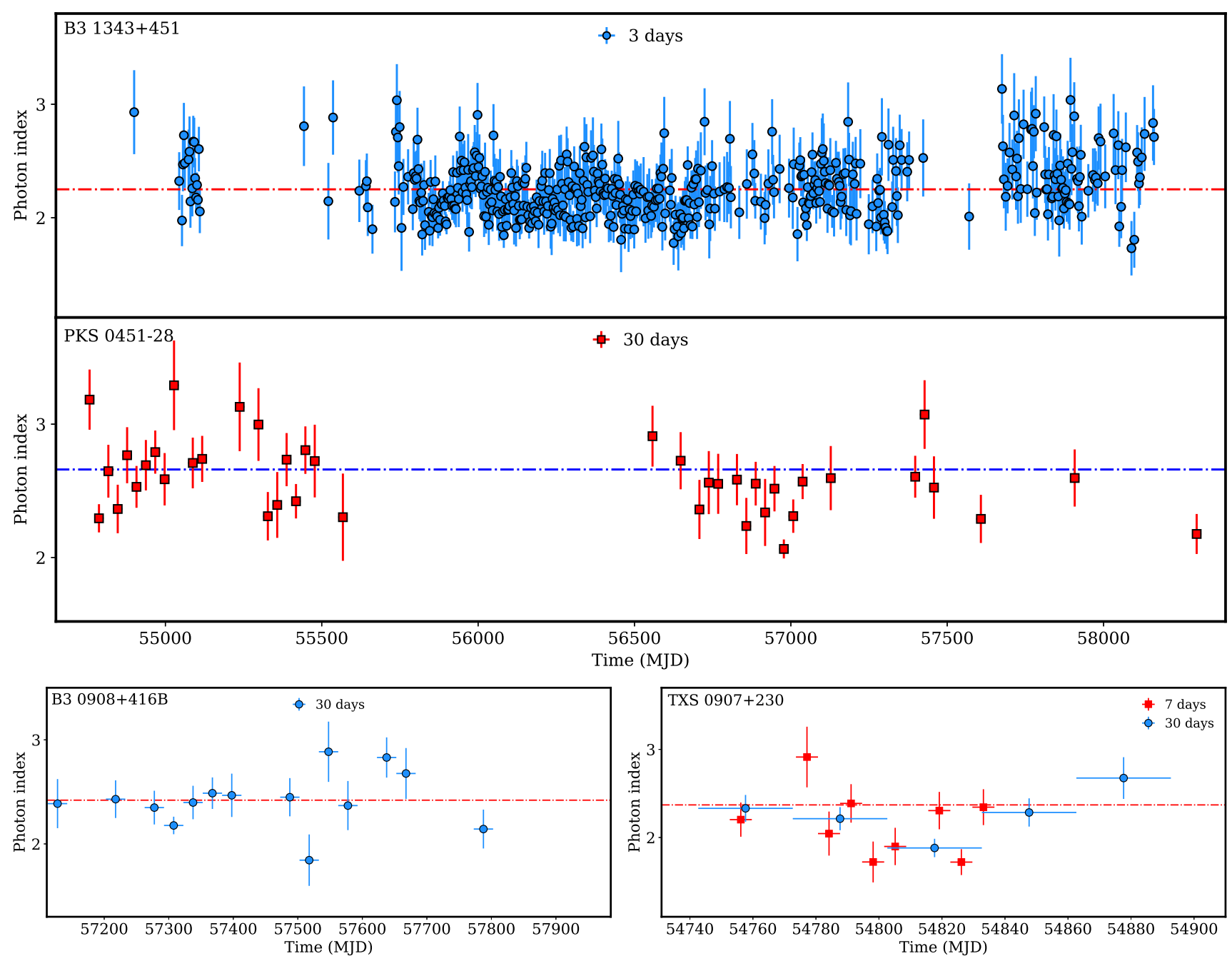

Figure 5. The evolution of the $\gamma$-ray photon indexes of B3 1343+451, PKS 0451-28, B3 0908+416B and TXS 0907+230 in time.

sidered sources, we have used a simple one-zone leptonic model. In this model, the emission region is assumed to be a spherical blob of radius of $R$ moving in the blazar jet with a bulk Lorentz factor of $\Gamma_{\mathrm{j}}$ at a viewing angle of $\theta$. The emitting region is filled with a uniformly tangled magnetic field $B$ and with a homogeneous population of relativistic electrons (and positrons), the nonthermal energy distribution of which is described by a power-law with an exponential cut-off at higher energies as:

$\mathrm{N}(\gamma) \sim \gamma^{-\alpha} \exp \left(-\gamma / \gamma_{\text {cut }}\right) \quad \gamma>\gamma_{\min }$

where $\gamma$ is the Lorentz factor of electrons in the blob rest frame, and $\alpha$ is the power-law index. $\gamma_{\min }$ and $\gamma_{\text {cut }}$ are the Lorentz factors corresponding to the minimum and cutoff energy of the electron distribution in the emission region. The total energy of the electrons in the emitting region is defined as $U_{\mathrm{e}}=m_{e} c^{2} \int \gamma N_{e}(\gamma) d \gamma$. The energy distribution of electrons given by Eq. 2 is formed when the emitting particles are accelerated with a limiting process at higher energies (e.g., cooling or limited efficiency of the acceleration process). The power-law index $(\alpha)$ defines the properties of the acceleration mechanism (e.g., Kirk \& Heavens 1989; Ellison et al. 1990; Summerlin \& Baring 2012; Drury 1983), and $\gamma_{\text {cut }}$ allows constraining the cooling process of the particles and the state of plasma in the jet (Baring et al. 2017; Sahakyan 2020).
In this interpretation, the parameters of the emitting region are the radius, Doppler factor ( $\Gamma=\delta$, for small viewing angles), and magnetic field. The magnetic field is a free parameter during the fitting (assuming its density $B^{2} / 8 \pi$ scales with $U_{\mathrm{e}}$ ), while in principle a constraint on the other two parameters can be derived from the data. For example, a lower limit on $\delta$ can be imposed using highresolution radio data or the emitting region size can be constrained from $R \leq \delta \times c \times t /(1+z)$, provided the minimum variability time is known. Unfortunately, both constraints cannot be assessed for all the sources given in Table 1, so both parameters have been left free during the fitting. In this case, $R$ is constrained from the observed Compton Dominance (CD- the IC to the synchrotron peak luminosities ratio) and from polynomial fitting of the data (see the documentation of jetset ${ }^{3}$ ).

In the modeling it is assumed that the low-energy peak (from radio to optical/UV) is due to synchrotron emission from ultrarelativistic electrons in the jet with an energy distribution as given by Eq. 2. Instead, the HE peak is due to the IC scattering of internal (SSC; Ghisellini et al. 1985; Bloom \& Marscher 1996; Maraschi et al. 1992) or external photons (EIC; Błażejowski et al.

\footnotetext{
${ }^{3}$ https://jetset.readthedocs.io/en/latest/
} 

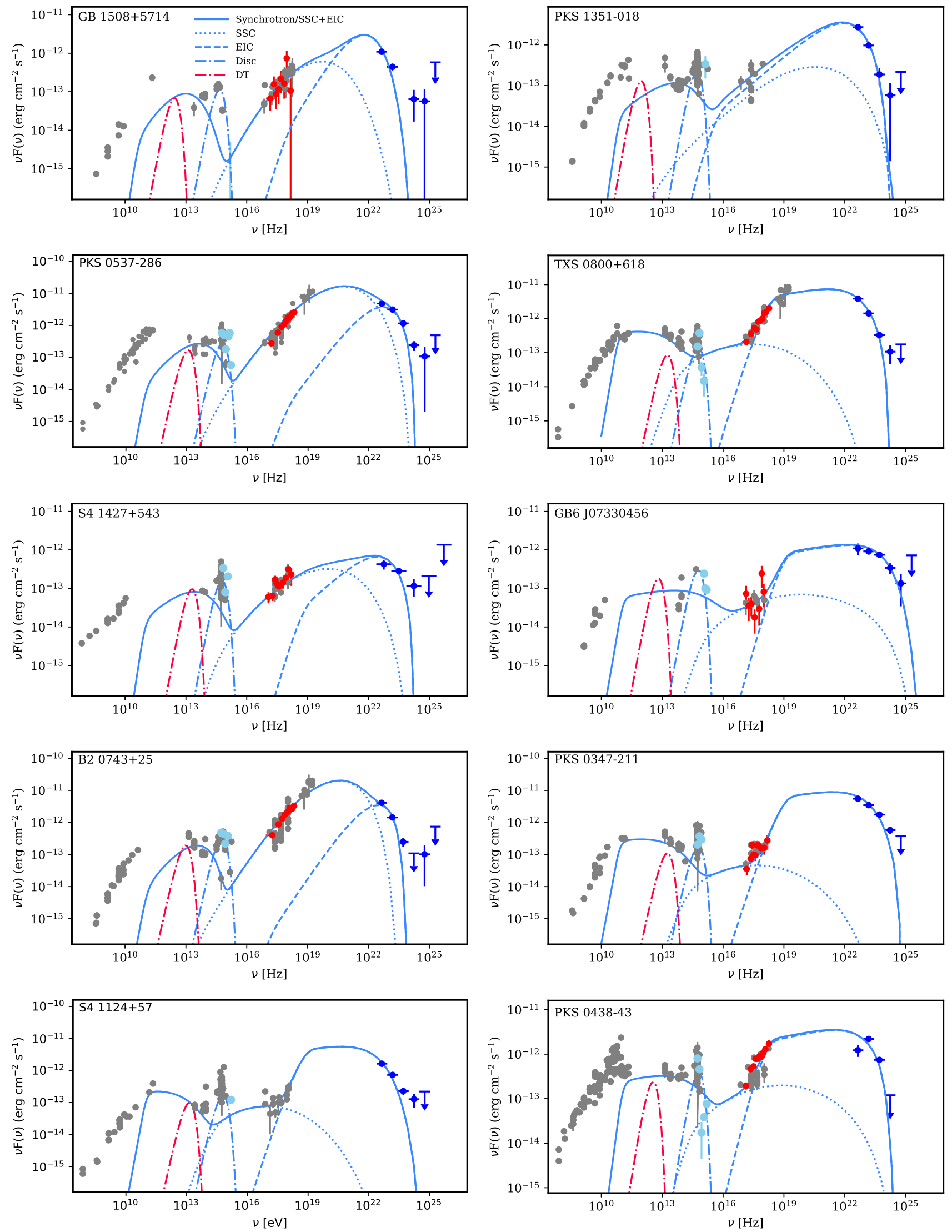

Figure 6. Modeling of the broadband SEDs of the considered sources. The Swift UVOT, XRT and Fermi-LAT data obtained here are shown with cyan, red and blue, respectively, while the archival data are in gray. 

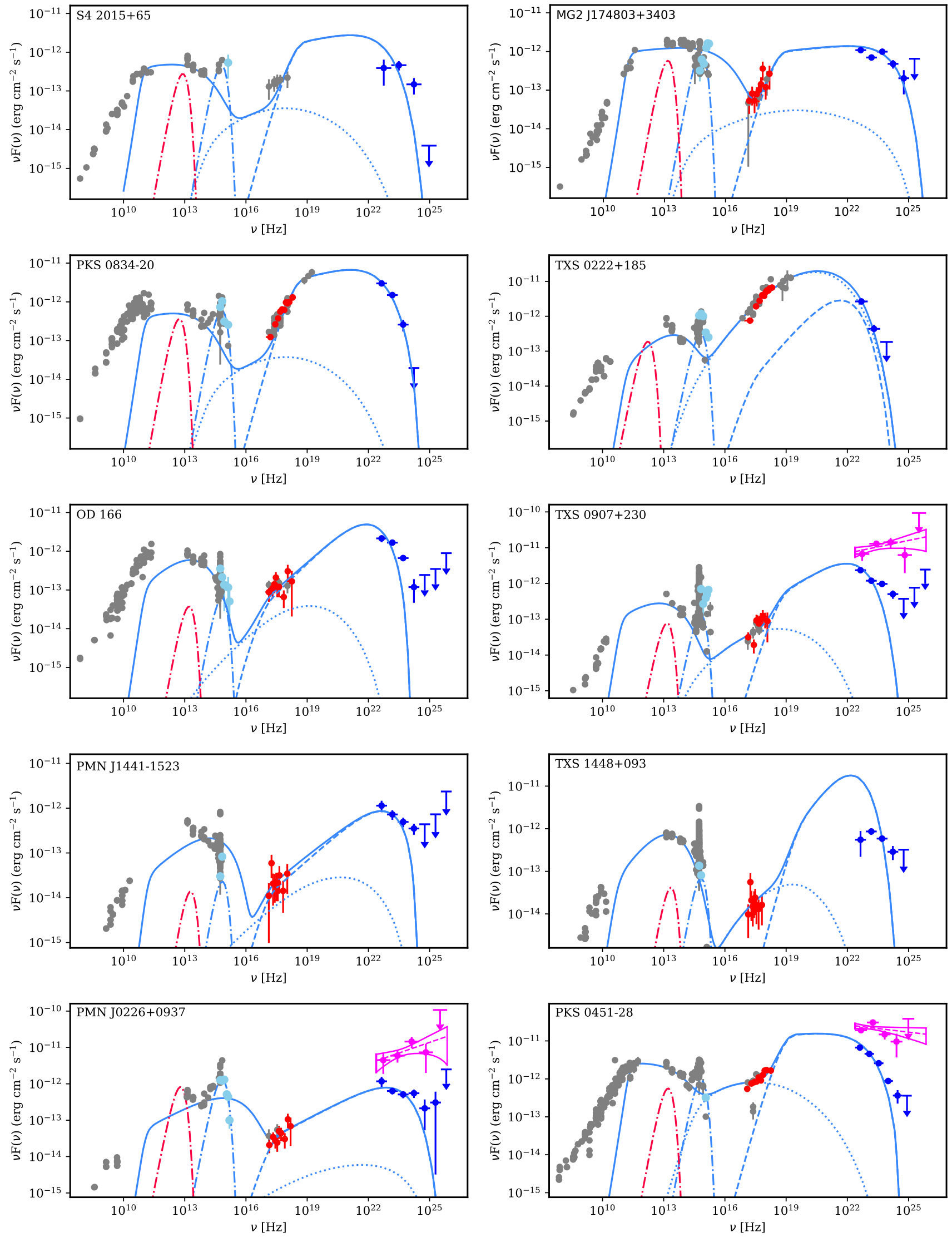

Figure 6. (Continued) 

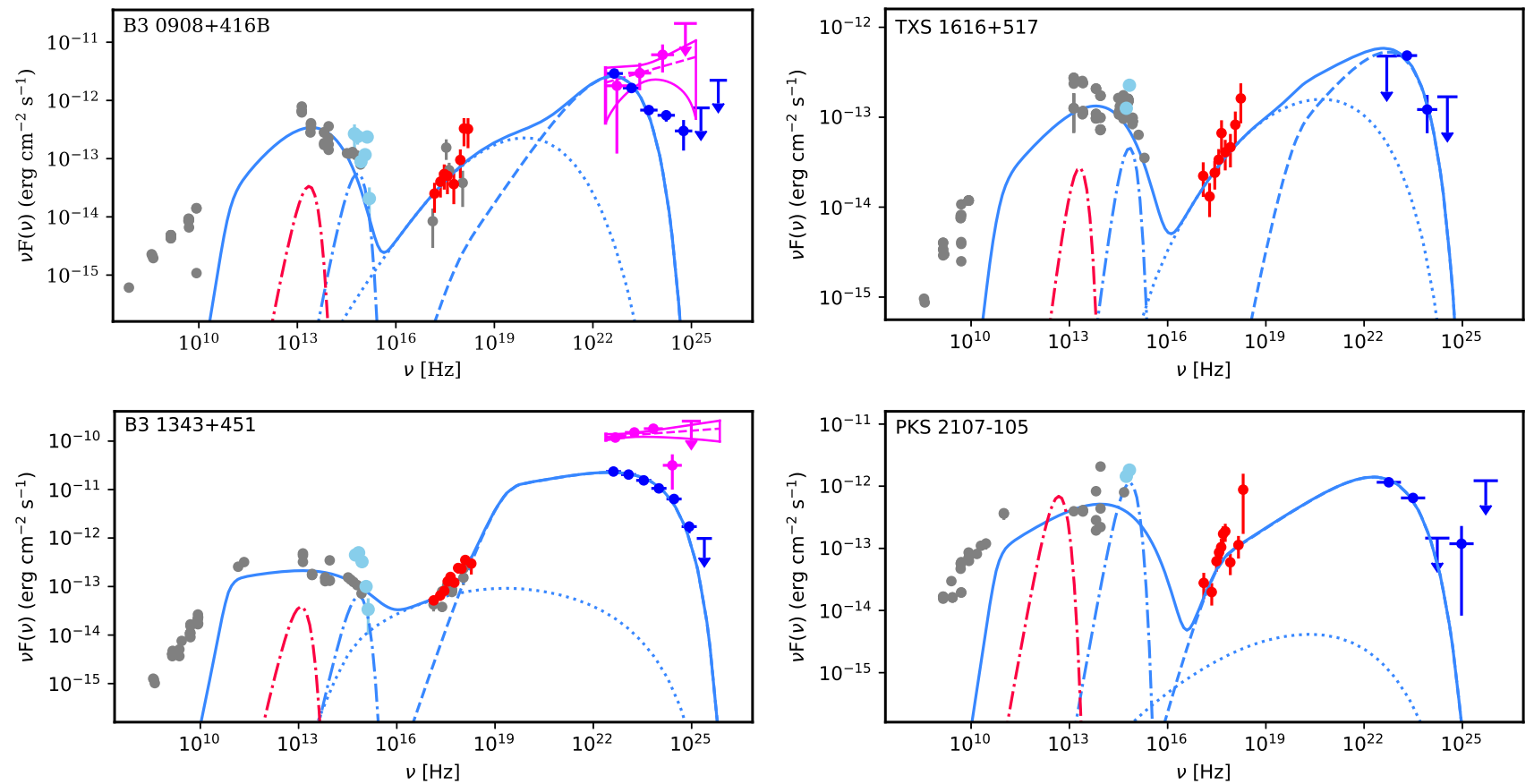

Figure 6. (Continued)

Table 5. Parameters obtained from the modeling of multiwavelength SEDs. [1]: object name. [2]: Doppler factor. [3]: Slope of electron energy distribution. [4] and [5]: the Lorentz factors corresponding to the minimum and cutoff energy of the electron distribution. [6]: Magnetic field in units of $G$. [7] Radius of the emitting region in units of $10^{16} \mathrm{~cm}$. [8] and [9]: Electron and magnetic field energy densities. [10]: accretion disk luminosity in units of 10 $0^{46}$ erg s ${ }^{-1}$. [11] and [12]: The power of the jet in the form of the relativistic electrons $\left(L_{e}\right)$ and magnetic field $\left(L_{B}\right)$ in units of $10^{45} \mathrm{erg} \mathrm{s}^{-1}$ and $10^{43} \mathrm{erg} \mathrm{s}^{-1}$, respectively.

\begin{tabular}{|c|c|c|c|c|c|c|c|c|c|c|c|}
\hline $\begin{array}{l}\text { Sources } \\
{[1]} \\
\end{array}$ & $\begin{array}{c}\delta \\
{[2]} \\
\end{array}$ & $\begin{array}{c}\alpha \\
{[3]} \\
\end{array}$ & $\begin{array}{c}\gamma_{\min } \\
{[4]}\end{array}$ & $\begin{array}{c}\gamma_{\text {cut }} \\
{[5]}\end{array}$ & $\begin{array}{c}B \\
{[6]} \\
\end{array}$ & $\begin{array}{c}R \\
{[7]} \\
\end{array}$ & $\begin{array}{l}U_{\mathrm{e}} \\
{[8]}\end{array}$ & $\begin{array}{l}U_{\mathrm{B}} \\
{[9]}\end{array}$ & $\begin{array}{c}L_{\mathrm{d}} \\
{[10]}\end{array}$ & $\begin{array}{c}L_{\mathrm{e}} \\
{[11]}\end{array}$ & $\begin{array}{c}L_{\mathrm{B}} \\
{[12]}\end{array}$ \\
\hline GB $1508+5714$ & $15.72 \pm 1.29$ & $1.17 \pm 0.07$ & $26.90 \pm 2.88$ & $1.30 \pm 0.10$ & $0.19 \pm 0.02$ & 2.28 & 0.50 & 1.49 & 3.02 & 2.43 & 0.73 \\
\hline PKS 1351-018 & $20.47 \pm 2.49$ & $2.16 \pm 0.11$ & $2.68 \pm 0.36$ & $4.99 \pm 0.71$ & $0.20 \pm 0.02$ & 2.29 & 0.54 & 1.66 & 4.04 & 2.68 & 0.82 \\
\hline PKS 0537-286 & $11.50 \pm 0.57$ & $1.33 \pm 0.07$ & $15.70 \pm 1.49$ & $2.45 \pm 0.16$ & $0.28 \pm 0.02$ & 1.14 & 5.93 & 3.21 & 3.44 & 7.31 & 0.40 \\
\hline TXS $0800+618$ & $14.04 \pm 0.56$ & $2.75 \pm 0.04$ & $13.98 \pm 0.86$ & $2.20 \pm 0.02$ & $0.26 \pm 0.01$ & 15.5 & 0.06 & 2.73 & 1.65 & 13.49 & 61.84 \\
\hline S4 1427+543 & $10.00 \pm 0.37$ & $2.04 \pm 0.10$ & $29.00 \pm 2.55$ & $2.79 \pm 0.27$ & $0.53 \pm 0.04$ & 1.90 & 0.63 & 10.02 & 1.83 & 2.14 & 3.75 \\
\hline GB6 J0733+0456 & $16.28 \pm 1.36$ & $2.80 \pm 0.04$ & $47.90 \pm 3.42$ & $15.73 \pm 1.60$ & $0.16 \pm 0.03$ & 2.98 & 0.12 & 6.22 & 3.40 & 0.98 & 5.20 \\
\hline B2 $0743+25$ & $10.02 \pm 0.45$ & $1.13 \pm 0.19$ & $7.66 \pm 0.22$ & $2.03 \pm 0.08$ & $0.36 \pm 0.01$ & 0.70 & 16.90 & 0.003 & 3.58 & 7.80 & 0.24 \\
\hline S4 1124+57 & $22.17 \pm 1.37$ & $2.78 \pm 0.04$ & $22.92 \pm 1.45$ & $1.28 \pm 0.10$ & $0.22 \pm 0.01$ & 5.15 & 0.14 & 1.95 & 1.69 & 3.39 & 4.87 \\
\hline PKS 0438-43 & $18.17 \pm 1.29$ & $2.78 \pm 0.04$ & $23.13 \pm 1.54$ & $7.19 \pm 0.58$ & $0.34 \pm 0.02$ & 5.42 & 0.12 & 4.52 & 3.91 & 3.33 & 12.52 \\
\hline S4 2015+65 & $17.85 \pm 1.32$ & $2.73 \pm 0.05$ & $20.63 \pm 1.64$ & $2.75 \pm 0.29$ & $0.46 \pm 0.03$ & 13.50 & 0.07 & 8.41 & 4.55 & 1.26 & 14.44 \\
\hline MG2 J174803+3403 & $24.50 \pm 2.06$ & $2.87 \pm 0.06$ & $14.67 \pm 1.58$ & $1.40 \pm 0.49$ & $1.45 \pm 0.09$ & 7.41 & 0.004 & 83.98 & 8.87 & 0.23 & 434.69 \\
\hline PKS 0834-20 & $27.42 \pm 0.97$ & $2.70 \pm 0.06$ & $20.57 \pm 1.51$ & $2.13 \pm 0.18$ & $0.37 \pm 0.02$ & 6.83 & 0.02 & 5.44 & 5.51 & 0.70 & 23.92 \\
\hline TXS $0222+185$ & $10.03 \pm 0.28$ & $1.62 \pm 0.05$ & $19.56 \pm 0.98$ & $2.38 \pm 0.10$ & $0.35 \pm 0.02$ & 1.07 & 10.28 & 5.04 & 2.71 & 11.10 & 0.54 \\
\hline OD 166 & $19.02 \pm 0.84$ & $1.96 \pm 0.04$ & $2.58 \pm 0.15$ & $1.01 \pm 0.03$ & $1.15 \pm 0.05$ & 4.32 & 0.01 & 0.52 & 0.53 & 0.25 & 92.38 \\
\hline PMN J1441-1523 & $17.01 \pm 1.50$ & $2.19 \pm 0.07$ & $2.86 \pm 0.29$ & $3.31 \pm 0.46$ & $1.68 \pm 0.14$ & 1.47 & 0.07 & 112.38 & 0.17 & 0.14 & 22.85 \\
\hline TXS 1448+093 & $17.90 \pm 1.13$ & $1.52 \pm 0.15$ & $49.64 \pm 5.82$ & $0.84 \pm 0.06$ & $0.70 \pm 0.05$ & 7.96 & 0.003 & 19.44 & 0.56 & 0.17 & 115.93 \\
\hline PMN J0226+0937 & $25.02 \pm 1.98$ & $2.41 \pm 0.04$ & $5.37 \pm 0.59$ & $8.32 \pm 0.62$ & $1.74 \pm 0.12$ & 3.09 & 0.03 & 119.86 & 10.94 & 0.03 & 107.98 \\
\hline PKS 0451-28 & $26.14 \pm 1.27$ & $2.90 \pm 0.28$ & $21.93 \pm 1.3^{\circ}$ & $2.19 \pm 0.01$ & $0.45 \pm 0.03$ & 5.90 & 0.11 & 8.01 & 7.20 & 3.59 & 26.32 \\
\hline B3 0908+416B & $23.22 \pm 1.72$ & $1.31 \pm 0.25$ & $6.41 \pm 0.72$ & $1.11 \pm 0.10$ & $0.39 \pm 0.03$ & 1.76 & 0.09 & 6.10 & 0.43 & 0.26 & 1.78 \\
\hline TXS 1616+517 & $10.11 \pm 0.31$ & $2.09 \pm 0.09$ & $93.28 \pm 4.15$ & $4.34 \pm 0.36$ & $0.52 \pm 0.02$ & 3.59 & 0.06 & 10.79 & 0.35 & 0.70 & 13.11 \\
\hline B3 $1343+451$ & $26.55 \pm 1.04$ & $2.48 \pm 0.04$ & $16.49 \pm 1.31$ & $8.67 \pm 0.48$ & $0.10 \pm 0.01$ & 4.16 & 0.11 & 0.42 & 0.48 & 1.76 & 0.68 \\
\hline PKS 2107-105 & $27.32 \pm 1.34$ & $2.30 \pm 0.06$ & $7.45 \pm 0.90$ & $3.63 \pm 0.39$ & $0.67 \pm 0.05$ & 9.48 & 0.0006 & 17.73 & 8.30 & 0.05 & 150.16 \\
\hline
\end{tabular}

2000; Sikora et al. 1994; Ghisellini \& Tavecchio 2009). The IC scattering of external photons is considered, since the SEDs of FSRQS are better explained by EIC, as shown by the previous studies (e.g., Ahnen et al. 2015; Abeysekara et al. 2015; MAGIC Collaboration et al. 2018; Hayashida et al. 2015b; Gasparyan et al. 2018), and the CD is evident in the SEDs of the considered sources (Fig. 6). Localization of the emission region in the jet is an open question and along the jet, depending on the distance from the central black hole, different photon fields can be dominant for the IC scattering (Sikora et al. 2009). In this paper we assume that the emitting region is outside the broad-line region (BLR) where the dominant photon field is the IR emission from the 
dusty torus. Sikora et al. (2002) showed that in MeV blazar SEDs the shift of the peak of the HE component to lower energies is most likely due to the comptonization of IR photons from the dusty torus. The IR radiation from the dusty torus is assumed to have a blackbody spectrum with a luminosity of $L_{\mathrm{IR}}=0.6 L_{\text {disc }}$ (see Ahnen et al. 2015) where $L_{\text {disc }}$ is the accretion disk luminosity, which fills a volume that for simplicity is approximated as a spherical shell with a radius of $R_{\mathrm{IR}}=2.5 \times 10^{18}\left(L_{\mathrm{d}} / 10^{45}\right)^{1 / 2} \mathrm{~cm}$ (Nenkova et al. 2008) with the energy density of $u_{\mathrm{IR}}=0.6 L_{\mathrm{d}} / 4 \pi R_{\mathrm{IR}}^{2} \delta^{2}$ in the co-moving frame of the jet. In Ghisellini et al. (2011) and Marcotulli et al. (2017), the HE component in the SED of distant blazars was modeled by IC scattering of BLR reflected photons, adopting a smooth broken power-law shape of the emitting electrons. We refer the reader to these papers for details on the modeling when BLR reflected photons are considered.

The broadband SEDs have been modeled using the jetset version 1.1.2 numerical leptonic code (Tramacere et al. 2011, 2009; Massaro et al. 2006). The free model parameters (those of the emitting electrons, $\delta, R$ and $B$ ) are constrained by using the Minuit optimizer. The emission directly due to accretion emerges primarily in the UV band, showing a UV excess in the SED, which is modeled by adding a blackbody component (Ghisellini et al. 2009b). Fitting of this excess allows us to estimate the disc photons temperature and luminosity. When the excess UV component is not distinguished, an upper limit is derived by requiring that the disc emission does not exceed the observed nonthermal emission from the jet.

\subsection{SED modeling results}

The SEDs modeling results are presented in Fig. 6 and the corresponding parameters in Table 5. The archival data from the Space Science Data Center ${ }^{4}$ are in gray, while the optical/UV, X-ray, and $\gamma$-ray data, obtained here, are shown in cyan, red, and blue, respectively. The radio data are not included in the SED fits but are considered only as upper limits. The observed radio emission is assumed to originate from a different and extended region. The hard $\gamma$-ray emission spectra of TXS 0907+230, PMN J0226+0937, PKS 0451-28, B3 0908+416B, and B3 1343+451 are in magenta, showing that the $\gamma$-ray flux increases and their spectra extend to higher energies. As high redshift blazars are considered $(z>2.5)$, their optical/UV flux could be affected by absorption of neutral hydrogen in intervening Lyman- $\alpha$ absorption systems. Following Ghisellini et al. (2011) this was corrected using the mean attenuation from Ghisellini et al. (2010b) which was computed for six wavelengths approximately centred in the UVOT filters.

The SEDs in Fig. 6 contain enough data from radio to HE $\gamma$-ray bands to shape both low and high energy peaks. The applied model reproduces the multiwavelength data relatively well for almost all the sources. The $\gamma$-ray data of TXS 1448+093 are not well explained by the model because in this case the optical/UV data are clearly constraining the HE tail of the electron distribution and if considering $\alpha>2.0$ (softer than the estimated spectrum with $\alpha=1.52 \pm 0.15$ ), the flux predicted by the model will be lower than the data. Due to the large uncertainties in the S4 2015+65 $\gamma$-ray data the modeling is difficult, so the best possible one is shown. The electron power-law index is defined by the X-ray data (through $\alpha=2 \Gamma_{\mathrm{X}}-1$ relation Rybicki \& Lightman 1986) and depending on whether the SSC or EIC component is dominating in the X-ray band, different values for $\alpha$ are obtained. When the $\mathrm{X}$-ray spectrum is hard

4 http://www.ssdc.asi.it and the SSC component is dominating, the energy distribution of the emitting electrons has a hard spectrum as well. For example, for GB 1508+5714, PKS 0537-286, B2 0743+25, TXS 0222+185 and B3 0908+416B, $\alpha=1.17 \pm 0.07,1.33 \pm 0.07,1.13 \pm 0.19$, $1.62 \pm 0.05$ and $1.31 \pm 0.25$ were estimated which shows that the emission is due to newly accelerated electrons. The X-ray emission from S4 $1427+543$ and TXS $1616+517$ is also dominated by the SSC component but because of large uncertainty in the X-ray photon index and flux estimations, correspondingly $\alpha=2.04 \pm 0.10$ and $2.09 \pm 0.09$ have been obtained. On the contrary, when the emission in the X-ray and $\gamma$-ray bands is only defined by the EIC component then $\alpha>2.2$; e.g., $\alpha=2.75 \pm 0.04$ and $2.70 \pm 0.06$ are correspondingly estimated for TXS 0800+618 and PKS 0834-20. Correspondingly, $\gamma_{\min }$ and $\gamma_{\text {cut }}$ are in the range of 2.58-93.28 and $(1.01-15.73) \times 10^{3}$ (excluding TXS 1448+093). The SSC bump in the X-ray band is sensitive to $\gamma_{\min }$ values (Ghisellini et al. 2009b; Tavecchio et al. 2000; Zhang et al. 2014) and those estimated here are well within the range usually estimated for the FSRQs (see Fig. 4 in Zhang et al. 2014). As expected (Celotti \& Ghisellini 2008), lower values of $\gamma_{\min }$ are estimated when the X-rays are produced only by the EIC component: e.g., $\gamma_{\min }=2.68 \pm 0.36$ and $2.58 \pm 0.15$ are correspondingly estimated for PKS 1351-018 and OD 166. The HE tails of both synchrotron and IC components are well defined by the optical/UV and $\gamma$-ray data, respectively, allowing precise estimation of $\gamma_{\text {cut }}=(1.01-15.73) \times 10^{3} \cdot \gamma_{\text {cut }}$ is in a strong dependence on $\alpha$, and its highest value, $(15.73 \pm 1.60) \times 10^{3}$ was estimated when $\alpha=2.80 \pm 0.04$ (the SED of GB6 J0733+0456 in Fig. 6). Meanwhile, when $\alpha=2.2-2.5$, the highest $\gamma_{\text {cut }}$ is $(8.67 \pm 0.48) \times 10^{3}$ for B3 1343+451.

The modeling shows that the magnetic field in the emitting region is within $0.10-1.74 \mathrm{G}$. The highest values of $1.74 \pm 0.12,1.68 \pm 0.14$, $1.45 \pm 0.09$ and $1.15 \pm 0.05 \mathrm{G}$ are estimated for PMN J0226+0937, PMN J1441-1523, MG2 J174803+3403, and OD 166, respectively. The estimated Doppler factor is from $\delta=10.00$ to $\delta=27.42$ with a mean of $\delta=19.09$. Although, these are higher than the average values estimated for FSRQs (e.g., Ghisellini \& Tavecchio 2015; Paliya et al. 2017), they are well within the range of physically realistic values (e.g., see Liodakis \& Pavlidou 2015). The emitting region size is within $R=(0.70-9.48) \times 10^{16} \mathrm{~cm}$ except for TXS $0800+618$ and S4 2015+65 for which $R=1.55 \times 10^{17} \mathrm{~cm}$ and $1.35 \times 10^{17} \mathrm{~cm}$, respectively. The values estimated for $R$ are consistent with the $\gamma$-ray flux variation in a day or several day scales and suggest that the multiwavelength emission is produced in the sub-parsec scale regions of the jet.

The energetics of the considered sources can be estimated using the modeling results. First, the available data allows a straightforward estimation of the disc luminosity of GB $1508+5714$, PKS 1351-108, PKS 0537-286, TXS 0800+618, S4 1427+543, GB6 J0733+0456, B2 0743+25, PKS 0347-211, S41124+57, PKS 043843, S4 2015+65, PKS 0834-20, TXS 0222+185, TXS 0907+230, PMN J0226+0937, PKS 0451-28 and PKS 2107-105, under the assumption that the disc has a black body spectrum. The estimation shows that $L_{\mathrm{d}} \simeq(1.09-10.94) \times 10^{46} \mathrm{erg} \mathrm{s}^{-1}$ with a highest value of $L_{\mathrm{d}} \simeq 1.09 \times 10^{47} \mathrm{erg} \mathrm{s}^{-1}$ estimated for PMN J0226+0937. Such high luminosities are obtained as very powerful blazars are considered here, and they are of the same order as those usually estimated for bright FSRQs (Pacciani et al. 2014) and distant blazars (Ghisellini et al. 2011; Paliya et al. 2016). The jet power in the form of the magnetic field $\left(L_{B}\right)$ and relativistic electrons $\left(L_{e}\right)$ is calculated as $L=\pi R^{2} c \Gamma^{2} U_{i}$, where $U_{i}$ is either electron $\left(U_{e}\right)$ or magnetic field $\left(U_{B}\right)$ energy density, using the parameters from Table 5 . The corresponding values are given in Table 5, showing that 
$L_{e}$ is in the range of $(0.03-13.49) \times 10^{45} \mathrm{erg} \mathrm{s}^{-1}$ while $L_{\mathrm{B}}$ in $(0.24-434.69) \times 10^{44} \mathrm{erg} \mathrm{s}^{-1} . L_{B}$ and $L_{e}$ are of the same order with $L_{\mathrm{e}} / L_{\mathrm{B}}=2.92$ and 0.61 for PKS 0834-20 and PMN J14411523 respectively, while for MG2 J174803+3403, OD 166, TXS 1448+093, PMN J0226+0937, PKS 2107-105 $L_{B} / L_{e}=3.7-36.0$. For the other sources of Table $5, L_{\mathrm{e}}$ exceeds $L_{\mathrm{B}}$, the largest deviation of $L_{\mathrm{e}} / L_{\mathrm{B}} \geq 1800$ being found for PKS 0537-286, B2 0743+25 and TXS $0222+185$, which are the only sources when the hard Xray emission above the Swift XRT band is modeled with a SSC component and the EIC is dominating at higher energies. However, we note that the luminosities can be higher when the SEDs in the flaring periods are modeled.

\section{DISCUSSION AND CONCLUSION}

Fermi-LAT has detected $\mathrm{MeV} / \mathrm{GeV}$ emission from 2500 blazars, which are bright emitters across the whole electromagnetic spectrum. Fermi-LAT has sufficient sensitivity to detect blazars farther than $z=2.0$, which are among the most powerful objects in the Universe. The distant objects $(z>2.0)$ represent a small fraction of the total observed sources $(3.75 \%)$, but their investigation is crucial for the study of the powerful relativistic outflows and measurement of the EBL photon density. We selected the most distant blazars $(z>2.5)$ from 4FGL and studied their multiwavelength emission properties by analyzing Fermi-LAT, Swift XRT and UVOT data. Also, the origin of their multiwavelength emission is investigated through theoretical modeling of the broadband SEDs.

In the X-ray and $\gamma$-ray bands, the spectra of the considered sources have different properties; except for TXS 1448+093, the X-ray spectrum of the other sources is hard with $\Gamma_{\mathrm{X}}=1.01-1.86$ while in the $\gamma$-ray band $\Gamma_{\gamma}>2.2$. Thus, the X-ray and $\gamma$-ray data are determining that the second peak in the SED is within $10^{6}-10^{8}$ $\mathrm{eV}$. The $\gamma$-ray flux of the considered sources is from $4.84 \times 10^{-10}$ to $1.50 \times 10^{-7}$ photon $\mathrm{cm}^{-2} \mathrm{~s}^{-1}$, and in the $\Gamma_{\gamma}-L_{\gamma}$ plane, they occupy the area more typical for bright blazars, which is natural, since the sources at large distances should be powerful enough to be detected. The two BL Lacs included in the sample, 87GB 214302.1+095227 and MG1 J154930+1708, are relatively faint, $\simeq(0.48-1.37) \times 10^{-9}$ photon $\mathrm{cm}^{-2} \mathrm{~s}^{-1}$, although it is already unusual to observe BL Lacs at large distances.

In the $F_{X}-\Gamma_{X}$ plane the considered sources occupy the region of hard $\mathrm{X}$-ray spectra $\left(\Gamma_{\mathrm{X}}<2.0\right)$ and a flux from $7.94 \times$ $10^{-14} \mathrm{erg} \mathrm{cm}^{-2} \mathrm{~s}^{-1}$ to $1.17 \times 10^{-11} \mathrm{erg} \mathrm{cm}^{-2} \mathrm{~s}^{-1}$. PKS 0451-28, TXS 0222+185, PKS 0834-20, PKS 0537- 286, TXS 0800+618, B2 $0743+25$ and PKS 0438-43 are separated from the others with a comparably high X-ray flux $F_{\mathrm{X} \text {-ray }} \geq 2.13 \times 10^{-12} \mathrm{erg} \mathrm{cm}^{-2} \mathrm{~s}^{-1}$. In the X-ray band, statistically significant flux variation is found for PKS 0438-43 and B2 $0743+25$ while there is an indication of variability for TXS $0222+185$; during the bright X-ray periods, their flux exceeds $10^{-11} \mathrm{erg} \mathrm{cm}^{-2} \mathrm{~s}^{-1}$.

The $\gamma$-ray flux variability can be investigated based on the available data. The most distant flaring blazars are MG3 J163554+3629 and PKS 0537-286 at $z=3.65$ and $z=3.10$, respectively. Though $\gamma$-ray flux amplification is observed in the 30-day bin light curve of MG3 J163554+3629, the $\gamma$-ray flares are more drastic and evident for PKS 0537-286. The adaptively binned light curve PKS 0537-286 shows several bright $\gamma$-ray flaring periods with a maximum flux of $(1.29 \pm 0.26) \times 10^{-6}$ photon $\mathrm{cm}^{-2} \mathrm{~s}^{-1}$ above 100 $\mathrm{MeV}$, observed on MJD 57879.2 in a time bin having a width of $\sim 16.0$ hours. For the distance of PKS 0537-286, such flux amplification can be useful for investigation of the $\gamma \gamma$ attenuation, but the photon index is $\Gamma_{\gamma}=2.64 \pm 0.27$, so the flare is dominated by sub-GeV photons. The $\gamma$-ray flux of B3 $1343+451$ increases in sub-day scales and that of PKS 0347-211 $(z=2.94)$ and PKS $0451-28(z=2.56)$ in day scales. B3 $1343+451$ is among the top 30 bright blazars observed by Fermi-LAT, showing multiple periods of enhanced $\gamma$-ray emission when the average luminosity of $(2-4) \times 10^{48} \mathrm{erg} \mathrm{s}^{-1}$ increased up to $1.5 \times 10^{50} \mathrm{erg} \mathrm{s}^{-1}$. The peak $\gamma$-ray flux of $(1.82 \pm 0.45) \times 10^{-6}$ photon $\mathrm{cm}^{-2} \mathrm{~s}^{-1}$ above $100 \mathrm{MeV}$ was observed on MJD 55891.7 which is $\geq 36.4$ times higher than the $\gamma$-ray flux in quiescent state. The $\chi 2$ test showed that the $\gamma$-ray emission of B3 0908+416B, TXS 0800+618, PKS 0438-43, OD 166 and TXS $0907+230$ is variable in week scales while that of MG3 J163554+3629, GB6 J0733+0456, B2 0743+25, PMN J1441-1523 and TXS $1616+517$ in month scales.

The $\gamma$-ray photon index of B3 1343+451, PKS 0451-28, B3 $0908+416 \mathrm{~B}$ and TXS $0907+230$ varies in time, and their $\gamma$-ray emission sometimes appears with a hard $\gamma$-ray spectrum. The averaged $\gamma$-ray photon index of these sources determines that the peak of the HE component to be below $10^{8} \mathrm{eV}$ whereas the hard $\gamma$-ray spectrum indicates that the peak is shifted to higher energies. During the flares, different processes can cause the shift of the low-energy or HE peaks. For example, both components will be shifted to HEs when the particles are effectively re-accelerated, resulting in higher electron cutoff energy $\left(\gamma_{\text {cut }}\right)$. It is expected that only the HE component will increase when due to the changes in the location of the emitting region the external photon fields are starting to dominate (Paggi et al. 2011). In order to understand the origin of the change in the emission components, extensive multiwavelength observations are required which is not the case for the sources considered here. We note that such changes have already been observed in the previous studies of blazars (e.g., Cutini et al. 2014; Ahnen et al. 2015; Abeysekara et al. 2015; Sahakyan \& Gasparyan 2017).

The main parameters characterizing the jets of the considered sources are derived by modeling the multiwavelength SEDs. The $\mathrm{CD}$ observed in the spectrum of almost all the considered sources implies that the electrons are loosing energy mainly by interacting with the external photons. In the framework of the single-zone scenario, the observed $\mathrm{X}$-ray and $\gamma$-ray data are satisfactorily explained taking into account IC scattering of synchrotron and IR photons which in its turn allows to constrain the parameters of the emitting electrons. The power-law index of the electrons is from $\sim 1.13$ to 2.90 , which is within the range expected from the standard particle acceleration theories. For example, in the diffuse shock particle acceleration, the formed particle spectra can be from very hard $(-1)$ to very steep, depending on the shock speed, nature of particle scattering, magnitude of turbulence, shock field obliquity, and other parameters (Summerlin \& Baring 2012). On the other hand, the optical/UV and $\gamma$-ray data strongly constrain the cut-off energy of electrons; the particles are effectively accelerated up to $\sim 10^{12} \mathrm{eV}$. The cooling of emitting particles defines the cut-off energy formed when the acceleration and cooling time scales are equal. Considering the electrons are mostly cooling by interacting with IR photons, and equating the radiative cooling time $t_{\text {cool }} \sim \frac{3 m_{e} c}{4 \sigma_{\mathrm{T}} u_{\mathrm{IR}}^{\prime} \gamma_{\mathrm{e}}^{\prime}}$ with the electron acceleration time $t_{\mathrm{acc}} \simeq \eta_{0} \frac{m_{e} c \gamma_{\mathrm{e}}^{\prime}}{e B}$ (Rieger et al. 2007), one gets that for $10^{12} \mathrm{eV}$ electrons these timescales are equal when $\eta^{-1}=5 \times 10^{-4}$. In other words, the maximum electron energy, if limited solely by the radiative energy losses, might be expected to be much higher than that observed, since often $\eta^{-1} \geq 10^{-2}$ is expected (e.g., Inoue \& Takahara 1996). Therefore the cutoff is most likely limited by the physical size of the emitting zone. For a given set of parameters, $R, B$, and $\delta, \gamma_{\min }$ is $<93$ and lower values of $\gamma_{\min }$ are 
estimated when the X-rays are produced only by EIC. This shows that the process responsible for the particle acceleration picks up almost all electrons.

The total jet luminosity (defined as $L=L_{e}+L_{B}$ ) is $\leq 1.41 \times$ $10^{46} \mathrm{erg} \mathrm{s}^{-1}$ which is of the same order as that usually calculated for blazars (Ghisellini et al. 2014). Thus, the jet power of distant and nearby blazars do not differ substantially. The estimated disc luminosity is within $L_{\mathrm{d}} \simeq(1.09-10.94) \times 10^{46} \mathrm{erg} \mathrm{s}^{-1}$, more typical for powerful blazars. The disc and jet luminosities are of the same order for TXS $0800+618$ and TXS $0222+185\left(L_{\text {disc }} / L_{\text {jet }}=1.17-2.44\right)$ while for the others $L_{\mathrm{disc}}$ is higher than $L_{\mathrm{jet}}$. However, we note that the protons with unknown content in jet are not included in the computation of $L_{\mathrm{jet}}$, so in principle higher $L_{\mathrm{jet}}$ are expected which could be of the same order as $L_{\text {disc }}$ (Maraschi \& Tavecchio 2003). Yet, when the accretion disc luminosity and temperature are well measured, an approximate value of the black hole mass can be derived following Ghisellini et al. (2009a, 2010a). In general, the black hole mass can be well estimated from the optical spectroscopy (Shaw et al. 2012) or from fitting the blue bump at the optical/UV band (Calderone et al. 2013; Ghisellini \& Tavecchio 2015). The maximum temperature (and hence the $v F v$ peak of the disc luminosity) of the standard multi-colour accretion disc temperature profile occurs at $5 R_{\mathrm{S}}$ where $R_{\mathrm{S}}$ is Schwarzschild radius. Taking into account that the peak temperature scales as $T_{\text {disc }} \sim\left(L_{\text {disc }} / R_{S}\right)^{1 / 4}$ (Ghisellini et al. 2009a), $R_{S}$ can be estimated from which the black hole mass can be derived. The black hole mass estimated for the sources with a clear blue-bump in their SED is in the narrow range of $(1.69-5.35) \times 10^{9} M_{\odot}$ where the highest black hole mass of $5.35 \times 10^{9} M_{\odot}$ is estimated for PMN J0226+0937 which has also the highest disc luminosity. The virial black hole mass of GB 1508+5714, PKS1351-018, S4 1427+543 and B2 $0743+25$ is also estimated in the quasar catalog of Shen et al. (2011) and is $(3.23 \pm 0.40) \times 10^{8} M_{\odot},(8.91 \pm 0.64) \times 10^{8} M_{\odot}$, $(1.80 \pm 0.17) \times 10^{8} M_{\odot}$ and $(3.89 \pm 0.26) \times 10^{9} M_{\odot}$, respectively. In the case of B2 $0743+25$, both methods produce similar results, $3.06 \times 10^{9} M_{\odot}$ and $(3.89-0.26) \times 10^{9} M_{\odot}$, while for the other sources the masses obtained using optical spectroscopy are slightly lower. Such differences are expected, since both methods rely on a fitting or approximations. For the considered sources, the Eddington luminosity is within $(2.12-5.87) \times 10^{48} \mathrm{erg} \mathrm{s}^{-1}$, and the ratio $L_{\mathrm{disc}} / L_{\mathrm{Edd}}$ ranges from 0.05 to 0.16 (e.g., see Ghisellini et al. 2014).

\section{SUMMARY}

The origin of the multiwavelength emission from distant blazars $(z>2.5)$ has been investigated using the Fermi-LAT data accumulated in 2008-2018 and Swift XRT/UVOT data observed in the last fifteen years. The main results are summarized as follows:

i) Twenty-six out of the thirty-three considered sources are FSRQs, five BCUs, and only two are BL Lacs. The two BL Lacs are also the faintest objects in the sample with a flux of $(0.48-$ 1.66) $\times 10^{-9}$ photon $\mathrm{cm}^{-2} \mathrm{~s}^{-1}$ ) while the others have a flux from $2.73 \times 10^{-9}$ photon $\mathrm{cm}^{-2} \mathrm{~s}^{-1}$ to $\sim 1.50 \times 10^{-7}$ photon $\mathrm{cm}^{-2} \mathrm{~s}^{-1}$.

ii) Except for the two BL Lacs, the photon index of all the considered sources ranges from 2.18 to 3.05. Only, the $\gamma$-ray indexes of B3 1343+451, PKS 0451-28, B3 0908+416B and TXS 0907+230 are found to vary in time. The hardest $\gamma$-ray spectra of B3 $1343+451$, B3 0908+416B and TXS 0907+230 are with indexes of $1.73 \pm 0.24$, $1.84 \pm 0.25$, and $1.72 \pm 0.15$, respectively, while that of PKS 0451-28 - with $2.06 \pm 0.07$. iii) The Swift XRT observations show a significant X-ray emission only from the FSRQs considered here. Only a few counts have been detected from the other sources, even if some of them have been observed by Swift several times. The X-ray photon index of distant FSRQs is $\Gamma_{\mathrm{X}}=1.1-1.8$ and the flux is spanning from $\sim 5 \times 10^{-14} \mathrm{erg} \mathrm{cm}^{-2} \mathrm{~s}^{-1}$ to $10^{-11} \mathrm{erg} \mathrm{cm}^{-2} \mathrm{~s}^{-1}$.

iv) The brightest X-ray source in the sample is TXS $0222+185$ $(z=2.69)$ with a flux of $(1.19 \pm 0.04) \times 10^{-11} \mathrm{erg} \mathrm{cm}^{-2} \mathrm{~s}^{-1}$. The X-ray emission from only PKS $0438-43$, B2 $0743+25$ and TXS $0222+185$ showed a substantial flux increase in some observations, whereas the X-ray emission from other sources is relatively constant in different years.

v) The $\gamma$-ray variability of the considered sources has been investigated using the data accumulated for ten years. Fourteen sources from the sample show a variable $\gamma$-ray emission on short and long timescales. The $\gamma$-ray flux of B3 $1343+451(z=2.53)$ and PKS 0537-286 $(z=3.10)$ increases in sub-day scales, that of PKS 0347$211(z=2.94)$ and PKS 0451-28 $(z=2.56)$ in day scales. The $\gamma$-ray emission of B3 0908+416B, TXS 0800+618, PKS 0438-43, OD 166 and TXS $0907+230$ is variable in a week scale while that of MG3 J163554+3629, GB6 J0733+0456, B2 0743+25, PMN J14411523 and TXS $1616+517$ in a month scale. The most distant $\gamma$-ray blazar flaring on short time scales is PKS 0537-286 $(z=3.10)$ when its flux increased up to $(1.29 \pm 0.26) \times 10^{-6}$ photon $\mathrm{cm}^{-2} \mathrm{~s}^{-1}$ above $100 \mathrm{MeV}$ within a time bin having a width of $\sim 16.0$ hours. The $\gamma$-ray flux of B3 1343+45 increased significantly from its average level in multiple periods with a maximum flux of $(1.82 \pm 0.45) \times 10^{-6}$ photon $\mathrm{cm}^{-2} \mathrm{~s}^{-1}$ above $100 \mathrm{MeV}$, accompanied by moderate hardening of the spectra.

vi) In the $\Gamma_{\gamma}-L_{\gamma}$ plane, the majority of the considered sources occupy the narrow range of $\Gamma_{\gamma}=2.2-3.1$ and $L_{\gamma}=(0.10-5.54) \times$ $10^{48} \mathrm{erg} \mathrm{s}^{-1}$, which is more typical for the brightest blazars. However, during $\gamma$-ray flares, the luminosity of variable sources is significantly beyond this boundary, changing within $\left(10^{49}-10^{50}\right) \mathrm{erg} \mathrm{s}^{-1}$. For example, the luminosity of B3 1343+451 increases $~ 36.4$ times and corresponds to $L_{\gamma}=1.5 \times 10^{50} \mathrm{erg} \mathrm{s}^{-1}$, being among the highest values so far observed in the $\gamma$-ray band.

vii) The SEDs were modeled within a one-zone leptonic scenario, considering the IC scattering of both synchrotron and IR photons from the dusty torus. The X-ray and $\gamma$-ray data allowed to constrain the peak of the HE component (within $10^{6}-10^{8} \mathrm{eV}$ ) as well as the power-law index and cut-off energy of the radiating electrons; the index and the cutoff energy are within the range of $1.13-2.90$ and $(1.01-15.73) \times 10^{3}$, respectively. The radius of the emitting region is estimated to be $\leq 0.05 \mathrm{pc}$ while the magnetic field and the Doppler factor are correspondingly within $0.10-1.74 \mathrm{G}$ and $10.00-27.42$.

viii) The jet luminosity is estimated to be $\leq 1.41 \times 10^{46} \mathrm{erg} \mathrm{s}^{-1}$, which is of the order of the values usually obtained for blazars. For all the sources, expect for TXS 0800+618 and TXS 0222+185 for which $L_{\mathrm{disc}} / L_{\text {jet }}=1.17-2.44$, the jet luminosity is lower than that of the disc $L_{\mathrm{d}} \simeq(1.09-10.94) \times 10^{46} \mathrm{erg} \mathrm{s}^{-1}$ which is estimated by fitting the UV excess. The black hole masses are estimated to be within $(1.69-5.35) \times 10^{9} M_{\odot}$ so the disc luminosity is $5-16 \%$ of the Eddington luminosity.

\section{ACKNOWLEDGEMENTS}

We thank the anonymous referee for constructive comments that improved the paper. This work was supported by the RA MoESCS Committee of Science, in the frames of the research project No 18T- 
1C335. This work used resources from the ASNET cloud and the EGI infrastructure with the dedicated support of CESGA (Spain). Part of this work is based on archival data, software or online services provided by the Space Science Data Center - ASI. This research has made use of the Swift XRT Data Analysis Software (XRTDAS). This work made use of data supplied by the UK Swift Science Data Centre at the University of Leicester.

\section{DATA AVAILABILITY}

The data underlying this article will be shared on reasonable request to the corresponding author.

\section{REFERENCES}

Abdo A. A., et al., 2010a, ApJ, 716, 30

Abdo A. A., et al., 2010b, ApJ, 722, 520

Abdo A. A., et al., 2010c, ApJ, 723, 1082

Abdollahi S., et al., 2020, ApJS, 247, 33

Abeysekara A. U., et al., 2015, ApJ, 815, L22

Ackermann M., et al., 2012, Science, 338, 1190

Ackermann M., Anantua R., et al. 2016, ApJ, 824, L20

Ackermann M., et al., 2017, ApJ, 837, L5

Ahnen M. L., et al., 2015, ApJ, 815, L23

Ajello M., et al., 2016, ApJ, 826, 76

Ajello M., et al., 2020, ApJ, 892, 105

Angioni R., Nesci R., Finke J. D., Buson S., Ciprini S., 2019, A\&A, 627, A140

Ansoldi S., et al., 2018, ApJ, 863, L10

Baghmanyan V., Gasparyan S., Sahakyan N., 2017, ApJ, 848, 111

Baring M. G., Böttcher M., Summerlin E. J., 2017, MNRAS, 464, 4875

Beall J. H., Bednarek W., 1999, ApJ, 510, 188

Bednarek W., Protheroe R. J., 1997, MNRAS, 287, L9

Błażejowski M., Sikora M., Moderski R., Madejski G. M., 2000, ApJ, 545,107

Bloemen H., et al., 1995, A\&A, 293, L1

Bloom S. D., Marscher A. P., 1996, ApJ, 461, 657

Böttcher M., Reimer A., Sweeney K., Prakash A., 2013, ApJ, 768, 54

Brown A. M., 2013, MNRAS, 431, 824

Burrows D. N., et al., 2005, Space Sci. Rev., 120, 165

Calderone G., Ghisellini G., Colpi M., Dotti M., 2013, MNRAS, 431, 210

Cash W., 1979, ApJ, 228, 939

Celotti A., Ghisellini G., 2008, MNRAS, 385, 283

Cerruti M., Zech A., Boisson C., Emery G., Inoue S., Lenain J.-P., 2019, MNRAS, 483, L12

Cheung C. C., 2017, The Astronomer's Telegram, 10356, 1

Cutini S., et al., 2014, MNRAS, 445, 4316

Dar A., Laor A., 1997, ApJ, 478, L5

Desai A., Helgason K., Ajello M., Paliya V., Domínguez A., Finke J., Hartmann D., 2019, ApJ, 874, L7

Domínguez A., et al., 2011, MNRAS, 410, 2556

Drury L. O., 1983, Reports on Progress in Physics, 46, 973

Dutka M. S., et al., 2017, ApJ, 835, 182

Ellison D. C., Jones F. C., Reynolds S. P., 1990, ApJ, 360, 702

Evans P. A., et al., 2009, MNRAS, 397, 1177

Fermi-LAT Collaboration et al., 2018, Science, 362, 1031

Foschini L., Ghisellini G., Tavecchio F., Bonnoli G., Stamerra A., 2011, A\&A, 530, A77

Foschini L., Bonnoli G., Ghisellini G., Tagliaferri G., Tavecchio F., Stamerra A., 2013, A\&A, 555, A138

Freedman W. L., et al., 2001, ApJ, 553, 47

Gao S., Fedynitch A., Winter W., Pohl M., 2019, Nature Astronomy, 3, 88

Gasparyan S., Sahakyan N., Baghmanyan V., Zargaryan D., 2018, ApJ, 863,114

Gehrels N., et al., 2004, ApJ, 611, 1005
Ghisellini G., Tavecchio F., 2009, MNRAS, 397, 985

Ghisellini G., Tavecchio F., 2015, MNRAS, 448, 1060

Ghisellini G., Maraschi L., Treves A., 1985, A\&A, 146, 204

Ghisellini G., Foschini L., Volonteri M., Ghirland a G., Haardt F., Burlon D., Tavecchio F., 2009a, MNRAS, 399, L24

Ghisellini G., Tavecchio F., Ghirlanda G., 2009b, MNRAS, 399, 2041

Ghisellini G., Tavecchio F., Foschini L., Ghirland a G., Maraschi L., Celotti A., 2010a, MNRAS, 402, 497

Ghisellini G., et al., 2010b, MNRAS, 405, 387

Ghisellini G., et al., 2011, MNRAS, 411, 901

Ghisellini G., Tavecchio F., Maraschi L., Celotti A., Sbarrato T., 2014, Nature, 515, 376

Hayashida M., Nalewajko K., et al. 2015a, ApJ, 807, 79

Hayashida M., et al., 2015b, ApJ, 807, 79

Inoue S., Takahara F., 1996, ApJ, 463, 555

Kaur A., et al., 2017, ApJ, 834, 41

Kaur A., Rau A., Ajello M., Domínguez A., Paliya V. S., Greiner J., Hartmann D. H., Schady P., 2018, ApJ, 859, 80

Keivani A., et al., 2018, ApJ, 864, 84

Kirk J. G., Heavens A. F., 1989, MNRAS, 239, 995

Li S., Xia Z.-Q., Liang Y.-F., Liao N.-H., Fan Y.-Z., 2018, ApJ, 853, 159

Liao N.-H., Xin Y.-L., Liang Y.-F., Guo X.-L., Li S., He H.-N., Yuan Q., Fan Y.-Z., 2018, preprint, (arXiv: 1807.05210)

Liodakis I., Pavlidou V., 2015, MNRAS, 454, 1767

Lott B., Escande L., Larsson S., Ballet J., 2012, A\&A, 544, A6

MAGIC Collaboration et al., 2018, A\&A, 619, A159

Mannheim K., 1993, A\&A, 269, 67

Mannheim K., 1995, Astroparticle Physics, 3, 295

Mannheim K., Biermann P. L., 1989, A\&A, 221, 211

Maraschi L., Tavecchio F., 2003, ApJ, 593, 667

Maraschi L., Ghisellini G., Celotti A., 1992, ApJ, 397, L5

Marcotulli L., et al., 2017, ApJ, 839, 96

Massaro E., Tramacere A., Perri M., Giommi P., Tosti G., 2006, A\&A, 448,861

Mattox J. R., et al., 1996, ApJ, 461, 396

Mücke A., Protheroe R. J., 2001, Astroparticle Physics, 15, 121

Mücke A., Protheroe R. J., Engel R., Rachen J. P., Stanev T., 2003, Astroparticle Physics, 18, 593

Nalewajko K., 2013, MNRAS, 430, 1324

Nenkova M., Sirocky M. M., Nikutta R., Ivezić Ž., Elitzur M., 2008, ApJ, 685,160

Pacciani L., Tavecchio F., Donnarumma I., Stamerra A., Carrasco L., Recillas E., Porras A., Uemura M., 2014, ApJ, 790, 45

Padovani P., Giommi P., 1995, ApJ, 444, 567

Padovani P., et al., 2017, A\&ARv, 25, 2

Paggi A., Cavaliere A., Vittorini V., D’Ammando F., Tavani M., 2011, ApJ, 736,128

Paliya V. S., 2015, ApJ, 804, 74

Paliya V. S., Parker M. L., Fabian A. C., Stalin C. S., 2016, ApJ, 825, 74

Paliya V. S., Marcotulli L., Ajello M., Joshi M., Sahayanathan S., Rao A. R., Hartmann D., 2017, ApJ, 851, 33

Paliya V. S., Cheung C. C. T., Kocevski D., Angioni R., 2018, The Astronomer's Telegram, 11847, 1

Paliya V. S., et al., 2019, ApJ, 871, 211

Poole T. S., et al., 2008, MNRAS, 383, 627

Rani B., Lott B., Krichbaum T. P., Fuhrmann L., Zensus J. A., 2013a, A\&A, 557, A71

Rani B., Lott B., Krichbaum T. P., Fuhrmann L., Zensus J. A., 2013b, A\&A, 557, A71

Rieger F. M., Bosch-Ramon V., Duffy P., 2007, Ap\&SS, 309, 119

Righi C., Tavecchio F., Inoue S., 2019, MNRAS, 483, L127

Roming P. W. A., et al., 2005, Space Sci. Rev., 120, 95

Rybicki G. B., Lightman A. P., 1986, Radiative Processes in Astrophysics

Sahakyan N., 2018, ApJ, 866, 109

Sahakyan N., 2019, A\&A, 622, A144

Sahakyan N., 2020, A\&A, 635, A25

Sahakyan N., Gasparyan S., 2017, MNRAS, 470, 2861

Sahakyan N., Baghmanyan V., Zargaryan D., 2018, A\&A, 614, A6 
Saito S., Stawarz L., et al. 2013, ApJ, 766, L11

Shakura N. I., Sunyaev R. A., 1973, A\&A, 500, 33

Shaw M. S., et al., 2012, ApJ, 748, 49

Shen Y., et al., 2011, ApJS, 194, 45

Sikora M., Begelman M. C., Rees M. J., 1994, ApJ, 421, 153

Sikora M., Błażejowski M., Moderski R., Madejski G. M., 2002, ApJ, 577,78

Sikora M., Stawarz L., Moderski R., Nalewajko K., Madejski G. M., 2009, ApJ, 704, 38

Stecker F. W., Malkan M. A., Scully S. T., 2006, ApJ, 648, 774

Summerlin E. J., Baring M. G., 2012, ApJ, 745, 63

Tavecchio F., Maraschi L., Sambruna R. M., Urry C. M., 2000, ApJ, 544, L23

Tramacere A., Giommi P., Perri M., Verrecchia F., Tosti G., 2009, A\&A, 501,879

Tramacere A., Massaro E., Taylor A. M., 2011, ApJ, 739, 66

Urry C. M., Padovani P., 1995, PASP, 107, 803

Volonteri M., 2010, A\&ARv, 18, 279

Wang K., Liu R.-Y., Li Z., Wang X.-Y., Dai Z.-G., 2018, preprint, (arXiv: 1809.00601)

Wright E. L., et al., 2010, AJ, 140, 1868

York D. G., et al., 2000, AJ, 120, 1579

Zargaryan D., Gasparyan S., Baghmanyan V., Sahakyan N., 2017, A\&A, 608, A37

Zhang J., Sun X.-N., Liang E.-W., Lu R.-J., Lu Y., Zhang S.-N., 2014, ApJ, 788, 104

This paper has been typeset from a $\mathrm{T}_{\mathrm{E}} \mathrm{X} / \mathrm{LT}_{\mathrm{E}} \mathrm{X}$ file prepared by the author. 